
\title{
R Research Soute \\ Required Time Gap Between Mainshock and Aftershock for Dynamic Analysis of Structures
}

\author{
Roohollah M. Pirooz
}

Kharazmi University

\section{Soheila Habashi}

Kharazmi University

Ali Massumi ( $\sim$ ali.massumi@gmail.com )

Kharazmi University https://orcid.org/0000-0003-4636-6553

\section{Research Article}

Keywords: time gap, seismic sequences, aftershock, zero acceleration interval, rest time

Posted Date: February 23rd, 2021

DOI: https://doi.org/10.21203/rs.3.rs-260285/v1

License: (c) (1) This work is licensed under a Creative Commons Attribution 4.0 International License.

Read Full License

Version of Record: A version of this preprint was published at Bulletin of Earthquake Engineering on April 2nd, 2021. See the published version at https://doi.org/10.1007/s10518-021-01087-z. 


\title{
Required time gap between mainshock and aftershock for dynamic analysis of structures
}

\author{
Roohollah M. Pirooz, Master graduate of Structural Engineering, Kharazmi University \\ Postal address: Kharazmi University, No.43, Dr. Mofatteh Ave., Tehran, IRAN \\ ORCID iD: 0000-0003-0761-9351
}

Soheila Habashi, Master graduate of Structural Engineering, Kharazmi University

Postal address: Kharazmi University, No.43, Dr. Mofatteh Ave., Tehran, IRAN

ORCID iD: 0000-0002-1631-2979

\author{
Ali Massumi, Professor of Structural Engineering, Kharazmi University \\ Postal address: Kharazmi University, No.43, Dr. Mofatteh Ave., Tehran, IRAN \\ ORCID iD: 0000-0003-4636-6553
}

Corresponding author: Ali Massumi

Email address: massumi@khu.ac.ir

Telephone: +982188830891

\begin{abstract}
Despite the various studies carried out to evaluate the effects of seismic sequences on structures, the matter of the time gap required to be considered between the mainshock and its corresponding aftershocks in dynamic analyses has never been focused on directly. This subtle but in the meantime effective subject, influences on the amount of accumulated damage caused by earthquake sequences. In the present study, 244 near fault ground motion components from 122 earthquakes were applied to a wide variety of single degree of freedom systems having vibrating period of 0.05 to 7 seconds with linear and nonlinear behavior. Furthermore, 2 planar steel moment-resisting frames, having 3 and 12 stories, were subjected to a set of 30 ground motion components. The purpose of this investigation was to estimate the required time for the structures to cease the free vibration at the end of the mainshock. The main purpose is to generate an estimation that is function of structural system's parameters and the strong motion duration. Excellent correlations were obtained between the rest time and the following parameters: the combination of natural period of single degree of freedom systems, as well as the strong motion duration of earthquake sequences. In consequence, a formula is proposed which estimates the required optimized rest-time of a structure based on natural vibration period, as well as the duration of strong motion. Additionally, results obtained from the dynamic analysis of the steel frames validate the rest-time values achieved from the proposed formula.
\end{abstract}

Keywords: time gap; seismic sequences; aftershock; zero acceleration interval; rest time

\section{Introduction}

The importance of aftershock effects on the responses of structures has been highlighted in several recent studies. The effect of successive earthquakes is of high importance not only for more accurate evaluation of framed structures and bridges (Xie et al. 2012) but also in maintaining the monuments and ancient structures (Papaloizou et al. 2016). Examples of seismic events followed by aftershocks of comparable intensity have been observed in various parts of the world, including in Italy (Friuli, 1976; Umbria-Marche, 1997), Greece (1986, 1988), Turkey (1999) and Mexico (1993, 1994, 1995) (Li and Ellingwood 2007). A key feature in the application of seismic sequences within the dynamic analysis of structures, is the time gap of a certain length inserted between the mainshock and the corresponding aftershocks. This silent time interval with zero acceleration is actually applied in 
dynamic analysis to ensure that the structure ceases the free vibration followed by the elimination of the seismic excitation at the end of the ground motion's time history (Goda 2012a, b).

In the real situation, usually aftershocks do not occur right after the mainshock time history is terminated and there is a time interval from a few minutes to several days between the mainshock and the subsequent aftershocks (P. Gallagher et al. 1996). The aforementioned time gap is an opportunity for the structure to stop vibration due to damping (Zhai et al. 2014). Amadio et al. (2003) considered a gap of about 40 seconds between successive events for the structure to stop moving (Amadio et al. 2003). Hatzigeorgiou et al. $(2009,2010)$ applied a time gap between the mainshock event and its following aftershocks which was equal to three times the single event duration (Hatzigeorgiou and Beskos 2009; Hatzigeorgiou and Liolios 2010). In other studies by Hatzigeorgiou (2010), a time gap of $100 \mathrm{~s}$ was applied between two consecutive seismic events (Hatzigeorgiou 2010a, b). Moustafa and Takewaki (2011) applied the 40 seconds separating time interval between events (Moustafa and Takewaki 2011).

Also, a silent time interval of 20 seconds was defined between the seismic sequence's component (Ruiz-García and Negrete-Manriquez 2011; Huang et al. 2012). Goda (2012) inserted 60 seconds of zeros, while in another study by Goda (2015), 30 seconds of zeros was added between the repeated earthquake time histories to ensure that the structural system returns to at-rest condition before the aftershock is applied (Goda 2012a, b, 2015). Zhai et al. (2014) considered a time gap of 100 seconds and claimed that this time gap is sufficient for any civil structure to stop vibrating due to damping (Zhai et al. 2013, 2014). Han et al. (2015) used different time gap for different mainshock-aftershock sequences (Han et al. 2015). Mirtaheri et al. (2017) applied the aftershock sequences immediately after the mainshock and claimed that the scenario could result in the most critical evaluation of their structural models (Mirtaherf et al. 2017). Pu and Wu (2018) considered a time interval of 15 times the fundamental period of structures employed for analysis ( $\mathrm{Pu}$ and $\mathrm{Wu}$ 2018). Hosseini et al. (2019) considered 200 seconds of zero acceleration time gap between selected sequences to ensure that the structure reaches the steady-state position (Hosseini et al. 2019). Damage-based yield point spectra were developed for repeated earthquake ground motions by analyzing single degree of freedom systems. In this work, a time gap of 100 seconds was considered between mainshock-aftershock sequences (Zhang et al. 2020). Considering 20 seconds time gap, seismic performance of buckling-restrained braced frames were evaluated under successive earthquakes (Hoveidae and Radpour 2020).

This study is presented to investigate the required time for the structures to stop the free vibration with respect to structural system's properties and the ground motion's features as the input parameters. Estimation of the minimum required time gap for the dynamic analyses based on the above-mentioned parameters is worth regarding since a wide variety of investigations conducted in earthquake engineering field to examine the effects of earthquake sequences on structures, do confront it. On the other hand, the cost of analyses is of a great importance especially in the repeated earthquake researches whereas the duration of seismic sequences is often long. Therefore, an optimized estimation of the required time gap is needed.

To this end, a formula is extracted using the curve fitting mathematical technique performed on the outputs of linear and nonlinear analyses of single degree of freedom systems. The influence of strain hardening, damping and ground motion scaling is taken into account in the proposed formulation. It is worth mentioning that results of two steel moment-resisting frames with 3 and 12 number of stories were examined to assure the validity and accuracy of the formulation.

\section{Theoretical background}

As mentioned earlier, the rest time between the mainshock and the aftershock is defined as the time in which the vibrating structure begins to enter the free vibration phase till the amplitude of vibration gradually is diminished and gets close to the zero value at the end. It is illustrated in Fig. 1 to better understand the definition of rest time.

Therefore, the velocity response of the structure obtained from the equation of motion when solved for the free vibration status after mainshock excitation, clarifies the rest time's influential parameters.

The dynamic equilibrium equation of motion for a single degree of freedom system is given in Eq. (1) (Chopra 2006):

$$
m \ddot{u}+c \dot{u}+k u=-m \ddot{u}_{g}
$$

where $\mathrm{m}$ is the mass of the system, $\mathrm{c}$ the viscous damping, $\mathrm{k}$ the stiffness, $\mathrm{u}$ the relative displacement, $\dot{u}$ the relative velocity, $\ddot{u}$ the relative acceleration and $\ddot{u}_{g}$ the ground motion acceleration. 


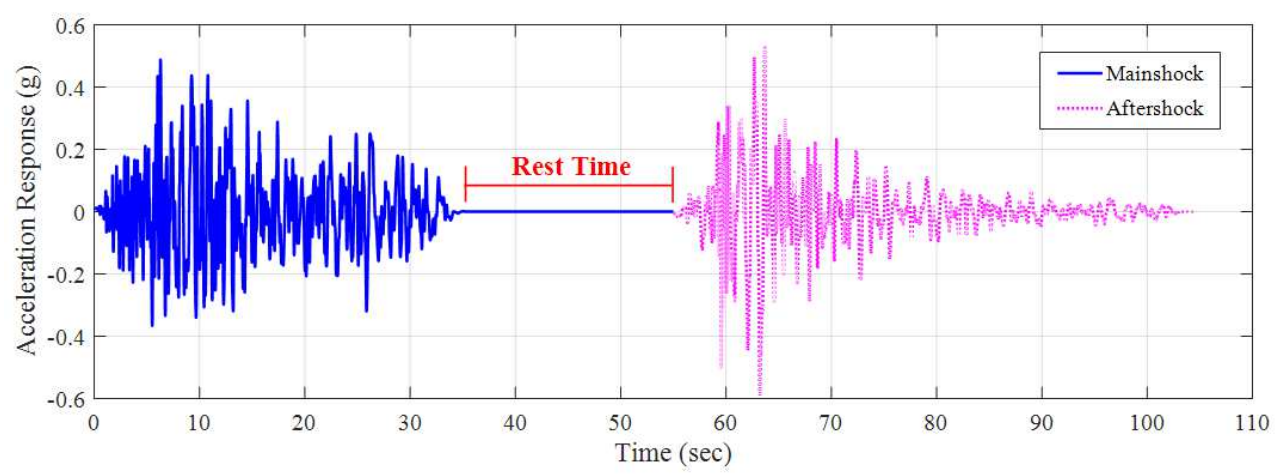

Fig. 1. An illustration of the rest time definition in a mainshock-aftershock earthquake record

If Eq. (1) is solved for a free vibrating system where the right term is set to zero, the general form of the velocity response will be evaluated as depicted in Fig. 2.

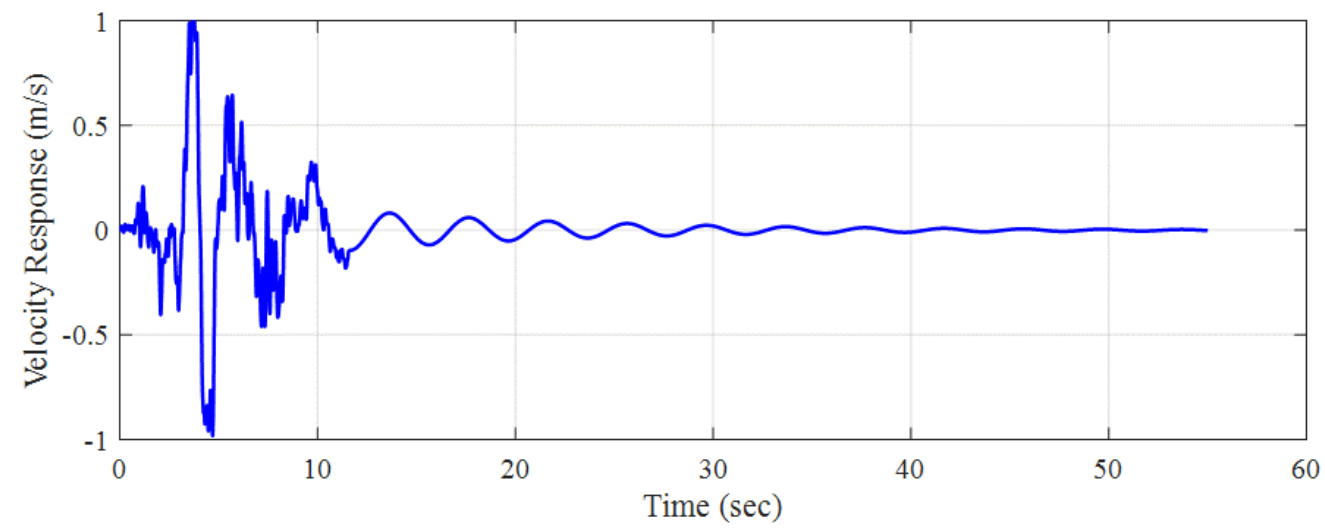

Fig. 2. General form of the velocity response of a SDOF system

From Fig. 2, it is evident that the amplitude of vibrational response reduces with time and finally drops to the value very close to zero. The time required for the velocity of the system to get close to zero, is a function of the system's damping ratio. The influence of damping on response of the system is also depicted in Fig. 3, in which the velocity response of a SDOF system considering two different damping ratios is compared.

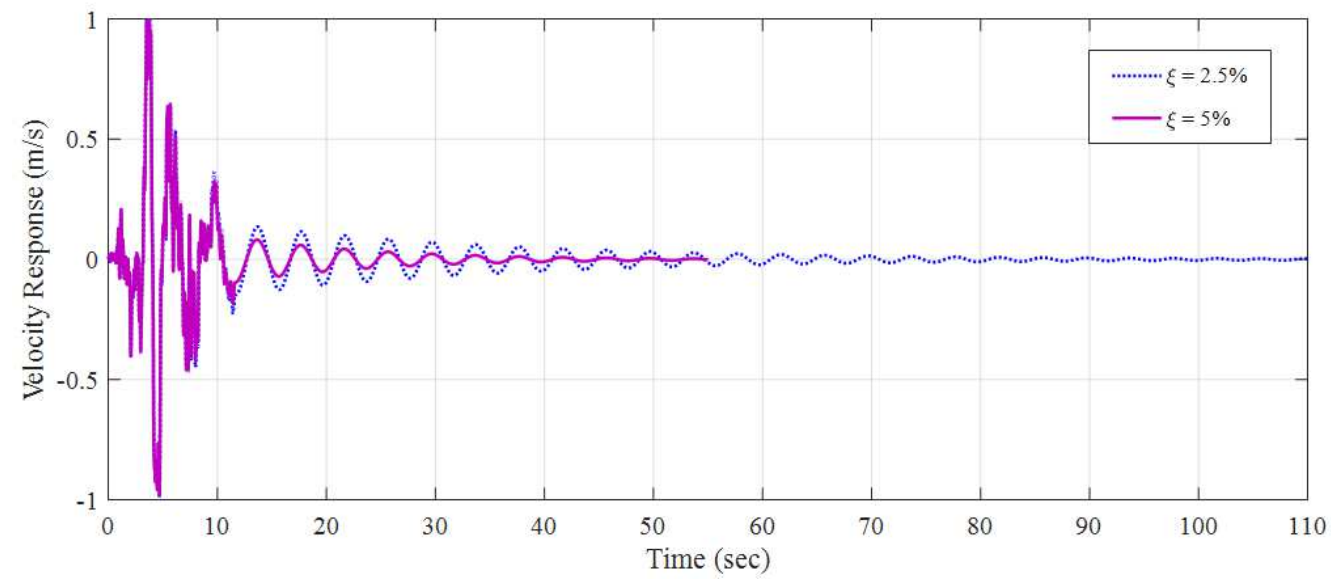

Fig. 3. Effect of damping ratio on the amplitude of velocity response and the required time for the response to be fully damped 
It is inferred that not only the amplitude of vibrational response is lessened as damping ratio is increased, but also the required time for the system response to be fully damped and get close to zero value is shortened drastically compared to the system with lower damping ratio $(\xi=2.5 \%)$. A similar trend for the MDOF systems applies except that mass $(\mathrm{m})$, stiffness $(\mathrm{k})$ and the damping coefficient (c) parameters are replaced by the mass, stiffness and damping matrices and the acceleration, velocity and the displacement responses $(\ddot{u}, \dot{u}, u)$ are replaced by their corresponding vectors in Eq. (1) (Far 2017).

\section{Description of structural models}

\subsection{Single degree of freedom models}

22 elastoplastic single degree of freedom (SDOF) systems with the vibration period of T, ranging from 0.05 to 7 seconds are used in this study (Table. 1). The nonlinear behavior of SDOF systems is defined by a bilinear elastoplastic force-displacement curve as shown in Fig. 4 (Samimifar et al. 2019). The equivalent bilinear forcedisplacement curves were obtained through pushover analysis carried out on framed structures as was recommended by Massumi and Monavari (2013) (Massumi and Monavari 2013). Strain-hardening ratio (h) or the ratio of postyielding stiffness to the initial stiffness of the model, is assumed to be $0 \%, 1 \%, 2 \%$ and $3 \%$. The ratio of ultimate to yield displacement (ductility) was set to 8 for all systems. Three values of $2.5 \%, 5 \%$ and $7.5 \%$ were also considered for viscous damping ratio $(\xi)$ of SDOF systems. Response of the models to ground motion acceleration time histories were computed using the Newmark $\beta=1 / 4$ numerical solution method of dynamic equation of motion (Clough and Penzien 1995). It is worth mentioning that soil-structure interaction is not required to be modeled for the assumed soil type (type II with shear velocity in range of $375-750 \mathrm{~m} / \mathrm{s}$ ) and conventional fixed base modeling is reliable (Tabatabaiefar et al. 2012).

Table 1. Fundamental period of vibration of single degree of freedom systems in seconds (T)

\begin{tabular}{lllllllllll}
\hline 0.05 & 0.10 & 0.15 & 0.20 & 0.25 & 0.50 & 0.75 & 1.00 & 1.25 & 1.50 & 1.75 \\
\hline 2.00 & 2.50 & 3.00 & 3.50 & 4.00 & 4.50 & 5.00 & 5.50 & 6.00 & 6.50 & 7.00 \\
\hline
\end{tabular}

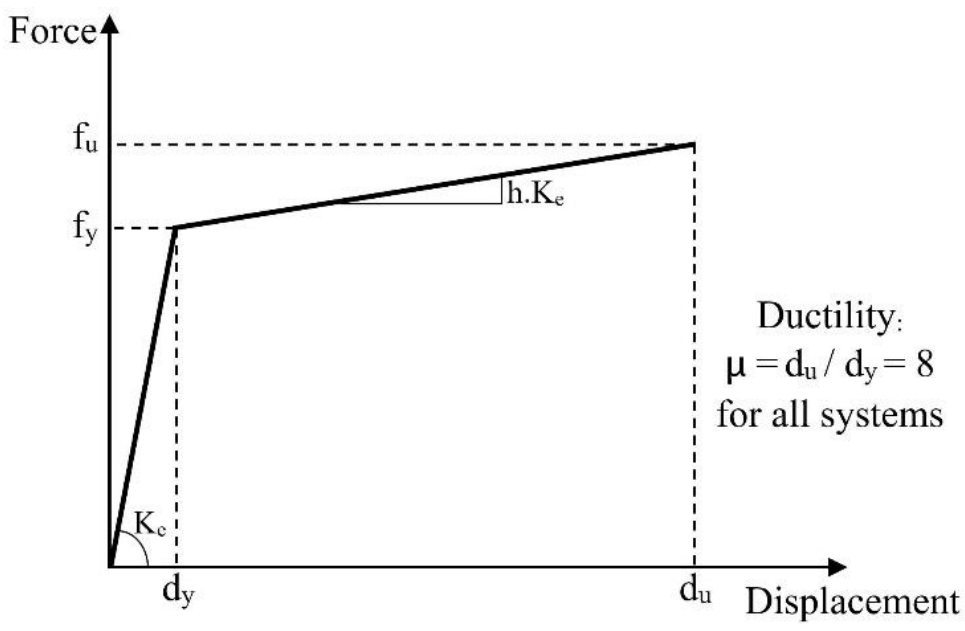

Fig. 4. Nonlinear behavior model of an SDOF system

\subsection{Multi degree of freedom models}

Two planar steel moment-resisting frames having 3 and 12 stories were modeled. The considered frames were designed according to provisions of AISC 360-16 (2016) (2016) and ASCE 7-16 (2017) (2017).

A more detailed description of the frame models is provided in Tables 2-3. The geometry and the elevation view of the frame models are also illustrated in Fig. 5.

The nonlinear behavior of columns and beams for dynamic analyses were assigned based on FEMA356 (Massumi and Mohammadi 2016) and is depicted in Fig. 6. 
Table 2. Properties of the frame models

\begin{tabular}{ccccc}
\hline $\begin{array}{c}\text { No. of } \\
\text { stories }\end{array}$ & $\begin{array}{c}\text { Story } \\
\text { height (m) }\end{array}$ & $\begin{array}{c}\text { No. of } \\
\text { bays }\end{array}$ & $\begin{array}{c}\text { Bay } \\
\text { width (m) }\end{array}$ & $\begin{array}{c}\text { Period of first } \\
\text { mode (sec.) }\end{array}$ \\
\hline 3 & 3.2 & 4 & 4 & 0.81 \\
\hline 12 & 3.2 & 4 & 4 & 1.98 \\
\hline
\end{tabular}

Table 3. Steel sections of the frame models

\begin{tabular}{cccc}
\hline Frame model & Story & Beams & Columns \\
\hline \multirow{3}{*}{3 Story } & 1,2 & IPE240 & BOX200X15 \\
\cline { 2 - 4 } & 3 & IPE200 & BOX200X15 \\
\hline \multirow{3}{*}{12 Story } & $1,2,3$ & IPE330 & BOX400X20 \\
\cline { 2 - 4 } & $4,5,6$ & IPE330 & BOX300X15 \\
\cline { 2 - 4 } & 10 & IPE300 & BOX250X15 \\
\cline { 2 - 4 } & 11 & IPE240 & BOX200X15 \\
\cline { 2 - 4 } & 12 & IPE200 & BOX200X15 \\
\hline
\end{tabular}

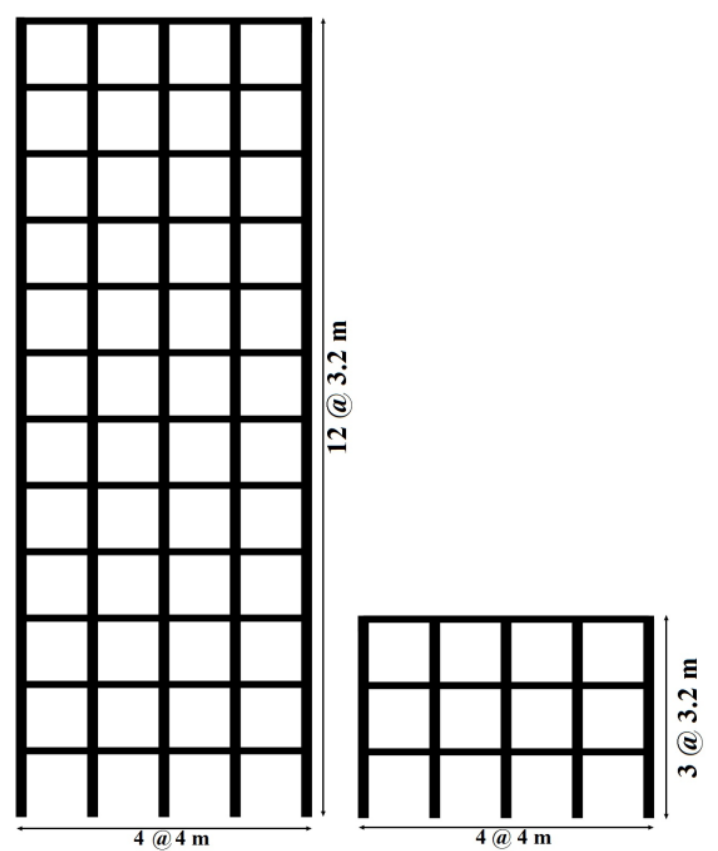

Fig. 5. Elevation model of the steel frames 


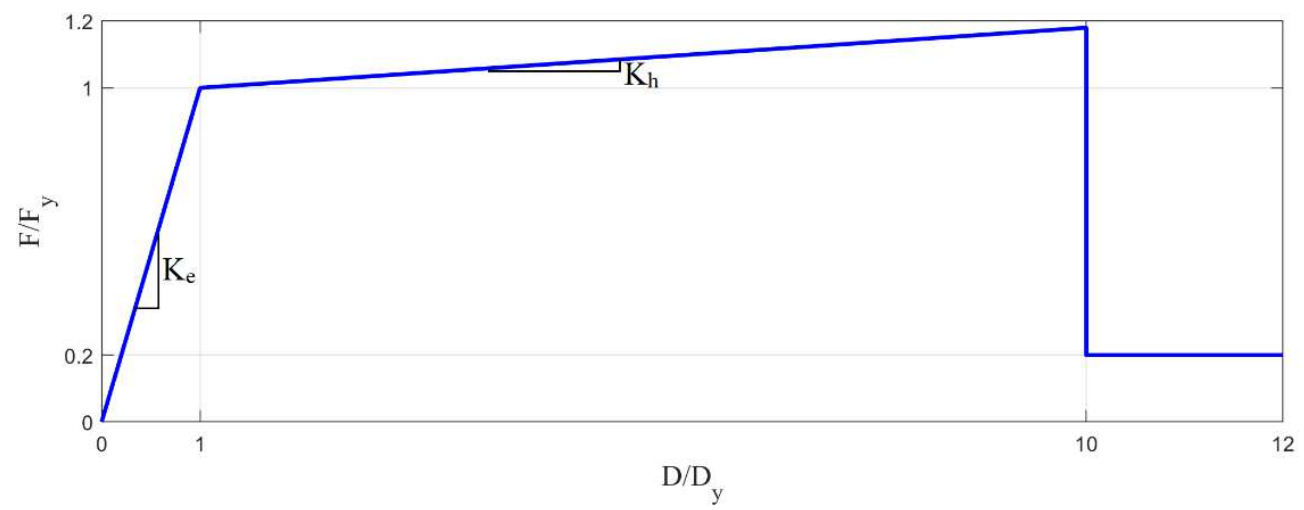

Fig. 6. Bilinear model of FEMA beam and column (D: end rotation or axial deformation, F: end moment or axial force, $\mathrm{K}_{\mathrm{e}}$ : initial elastic slope, $\mathrm{K}_{\mathrm{h}}$ : strain hardening slope, $\mathrm{K}_{\mathrm{h}} / \mathrm{K}_{\mathrm{e}}=0.02$ )

\section{Description of selected earthquake ground motions}

In this study, a total of 244 earthquake acceleration time histories from 122 earthquake events (longitudinal (LN) and transverse (TR) components) were employed for the dynamic analysis of the SDOF systems and a set of 30 acceleration time histories of the aforementioned ensemble were selected for the analysis of the steel frames. During selection of records it is tried to include a wide variety of peak ground acceleration (PGA), peak ground velocity (PGV), PGA/PGV ratio as a simple indicator of frequency content (Zhu et al. 1988) and strong motion duration $\left(T_{d}\right)$ as the most important parameters on dynamic response of structures. Fig. 7 depicts the aforementioned parameters of selected earthquake ground motions.
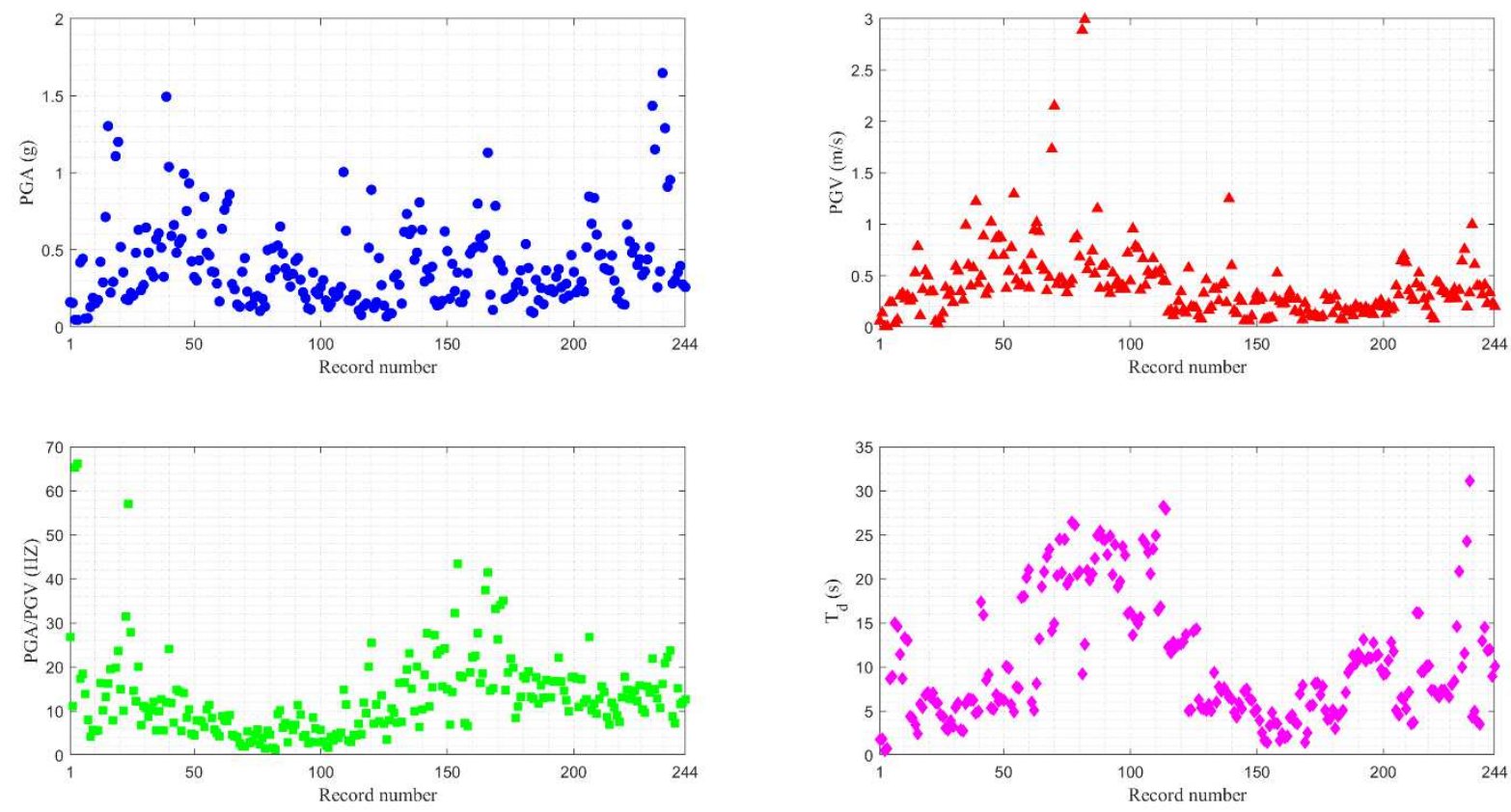

Fig. 7. Descriptor parameters of selected records

All the earthquake ground motions were downloaded from the Pacific Earthquake Engineering Research (PEER) center database. Regarded earthquake records were selected based on the following criteria: (i) Magnitude $\left(M_{w}\right)$ of the ground motions varies between 6 and 7.62; (ii) The shortest distance from the site to the rupture surface $\left(R_{\text {rup }}\right)$ is less than or equal to 10 kilometers (i.e., the selected records are near fault events); (iii) The average shear wave velocity of top $30 \mathrm{~m}$ of the site $\left(V_{s 30}\right)$ which corresponds to the soil type and site condition varies between $375 \mathrm{~m} / \mathrm{s}$ and $750 \mathrm{~m} / \mathrm{s}$ conforming to site class C according to ASCE site classification. 
The list of the considered earthquakes is shown in Table 4. Ground motions which were utilized for the analyses of steel frames are highlighted and signed with star mark. The term $T_{d}$ in last column of the Table 4 , indicates the interval of 5-95\% of squared ground acceleration cumulative integral which is identified as the most suitable duration metric (Deierlein et al. 2012).

Table 4. Earthquake ground motions employed for the analyses of SDOF systems

\begin{tabular}{|c|c|c|c|c|c|c|c|c|c|c|c|}
\hline \multirow{2}{*}{ No. } & \multirow{2}{*}{$\begin{array}{l}\text { Earthquak } \\
\text { e Name }\end{array}$} & \multirow{2}{*}{ Station Name } & \multirow{2}{*}{ Mw } & \multirow{2}{*}{$\begin{array}{l}\mathbf{R}_{\text {rup }} \\
(\mathbf{k m})\end{array}$} & \multirow{2}{*}{$\begin{array}{l}\mathbf{V}_{\mathrm{s30}} \\
(\mathrm{m} / \mathbf{s})\end{array}$} & \multicolumn{2}{|c|}{ PGA (g) } & \multicolumn{2}{|c|}{ PGA/PGV (Hz) } & \multicolumn{2}{|c|}{$\mathbf{T}_{\mathrm{d}}(\mathrm{sec})$} \\
\hline & & & & & & $\mathbf{L N}$ & TR & $\mathbf{L N}$ & TR & $\mathbf{L N}$ & TR \\
\hline 1 & $\begin{array}{c}\text { Helena, } \\
\text { Montana-01 }\end{array}$ & Carroll College & 6 & 2.86 & 593.35 & 0.16 & 0.16 & 26.73 & 11.40 & 1.74 & 1.82 \\
\hline 2 & $\begin{array}{c}\text { Helena, } \\
\text { Montana-02 }\end{array}$ & Helena Fed Bldg & 6 & 2.92 & 551.82 & 0.05 & 0.05 & 68.10 & 73.18 & 0.49 & 0.76 \\
\hline 3 & Morgan Hill & $\begin{array}{c}\text { Anderson Dam } \\
\text { (Downstream) }\end{array}$ & 6.19 & 3.26 & 488.77 & 0.42 & 0.29 & 16.22 & 10.23 & 4.385 & 4.205 \\
\hline 4 & Morgan Hill & $\begin{array}{c}\text { Coyote Lake Dam - } \\
\text { Southwest } \\
\text { Abutment } \\
\end{array}$ & 6.19 & 0.53 & 561.43 & 0.71 & 1.3 & 13.17 & 16.26 & 3.425 & 2.41 \\
\hline 5 & Morgan Hill & Gilroy Array \#6 & 6.19 & 9.87 & 663.31 & 0.22 & 0.29 & 19.28 & 7.80 & 5.79 & 5.415 \\
\hline 6 & Big Bear-01 & $\begin{array}{l}\text { Big Bear Lake - } \\
\text { Civic Center }\end{array}$ & 6.46 & 8.3 & 430.36 & 0.48 & 0.54 & 14.84 & 14.28 & 8.52 & 9.18 \\
\hline 7 & Kobe, Japan & Nishi-Akashi & 6.9 & 7.08 & 609 & 0.48 & 0.46 & 10.29 & 11.26 & 7.72 & 7.56 \\
\hline 8 & $\begin{array}{l}\text { Duzce, } \\
\text { Turkey } \\
\end{array}$ & Lamont 1058 & 7.14 & 0.21 & 529.18 & 0.11 & 0.08 & 7.35 & 4.83 & 12.28 & 11.61 \\
\hline 9 & $\begin{array}{l}\text { Duzce, } \\
\text { Turkey }\end{array}$ & Lamont 1059 & 7.14 & 4.17 & 551.3 & 0.14 & 0.15 & 12.16 & 9.40 & 12.81 & 12.24 \\
\hline 10 & $\begin{array}{l}\text { Duzce, } \\
\text { Turkey }\end{array}$ & Lamont 375 & 7.14 & 3.93 & 454.2 & 0.51 & 0.89 & 19.92 & 25.45 & 12.54 & 12.65 \\
\hline 11 & $\begin{array}{l}\text { Duzce, } \\
\text { Turkey }\end{array}$ & Lamont 531 & 7.14 & 8.03 & 638.39 & 0.12 & 0.16 & 6.93 & 11.45 & 12.87 & 13.65 \\
\hline 12 & $\begin{array}{l}\text { Chi-Chi, } \\
\text { Taiwan-04 }\end{array}$ & CHY074 & 6.2 & 6.2 & 553.43 & 0.32 & 0.34 & 9.53 & 7.27 & 5.235 & 5.06 \\
\hline 13 & Tottori, Japan & SMN015 & 6.61 & 9.12 & 616.55 & 0.27 & 0.15 & 16.19 & 7.49 & 5.75 & 5.04 \\
\hline 14 & Tottori, Japan & SMNH01 & 6.61 & 5.86 & 446.34 & 0.62 & 0.73 & 16.52 & 19.36 & 9.39 & 6.01 \\
\hline 15 & Tottori, Japan & TTR009 & 6.61 & 8.83 & 420.2 & 0.6 & 0.63 & 22.98 & 15.09 & 7.77 & 7.25 \\
\hline 16 & Bam, Iran & Bam & 6.6 & 1.7 & 487.4 & 0.81 & 0.63 & 6.36 & 10.36 & 6.565 & 6.33 \\
\hline 17 & $\begin{array}{l}\text { Parkfield-02, } \\
\text { CA }\end{array}$ & $\begin{array}{l}\text { PARKFIELD - } \\
\text { DONNA LEE }\end{array}$ & 6 & 4.93 & 656.75 & 0.29 & 0.37 & 17.89 & 27.44 & 4.845 & 4.33 \\
\hline 18 & $\begin{array}{l}\text { Parkfield-02, } \\
\text { CA }\end{array}$ & $\begin{array}{l}\text { PARKFIELD - } \\
\text { EADES }\end{array}$ & 6 & 2.85 & 383.9 & 0.32 & 0.39 & 11.09 & 15.33 & 5.885 & 5.27 \\
\hline 19 & $\begin{array}{l}\text { Parkfield-02, } \\
\text { CA }\end{array}$ & $\begin{array}{c}\text { PARKFIELD - } \\
\text { GOLD HILL }\end{array}$ & 6 & 3.43 & 558.33 & 0.17 & 0.14 & 27.11 & 22.96 & 7.255 & 7.475 \\
\hline 20 & $\begin{array}{l}\text { Parkfield-02, } \\
\text { CA }\end{array}$ & $\begin{array}{l}\text { PARKFIELD - } \\
\text { JACK CANYON }\end{array}$ & 6 & 9.46 & 576.21 & 0.15 & 0.17 & 24.31 & 15.57 & 6.41 & 6.245 \\
\hline 21 & $\begin{array}{l}\text { Parkfield-02, } \\
\text { CA }\end{array}$ & $\begin{array}{c}\text { PARKFIELD - } \\
\text { JOAQUIN } \\
\text { CANYON } \\
\end{array}$ & 6 & 4.57 & 378.99 & 0.62 & 0.49 & 24.18 & 14.91 & 4.905 & 5.165 \\
\hline 22 & $\begin{array}{l}\text { Parkfield-02, } \\
\text { CA }\end{array}$ & $\begin{array}{l}\text { PARKFIELD - } \\
\text { MIDDLE } \\
\text { MOUNTAIN } \\
\end{array}$ & 6 & 2.57 & 397.57 & 0.18 & 0.41 & 6.73 & 14.34 & 3.945 & 2.52 \\
\hline 23 & $\begin{array}{l}\text { Parkfield-02, } \\
\text { CA }\end{array}$ & $\begin{array}{c}\text { PARKFIELD - } \\
\text { STOCKDALE } \\
\text { MTN }\end{array}$ & 6 & 4.83 & 393.56 & 0.23 & 0.35 & 31.77 & 42.95 & 1.67 & 1.465 \\
\hline 24 & $\begin{array}{l}\text { Parkfield-02, } \\
\text { CA }\end{array}$ & $\begin{array}{l}\text { Bear Valley Ranch, } \\
\text { Parkfield, CA, USA }\end{array}$ & 6 & 4.32 & 527.95 & 0.16 & 0.16 & 17.61 & 17.55 & 3.35 & 4.7875 \\
\hline 25 & $\begin{array}{c}\text { Parkfield-02, } \\
\text { CA } \\
\end{array}$ & Slack Canyon & 6 & 2.99 & 648.09 & 0.21 & 0.35 & 7.24 & 6.54 & 3.675 & 3.56 \\
\hline 26 & $\begin{array}{l}\text { Parkfield-02, } \\
\text { CA }\end{array}$ & $\begin{array}{c}\text { Parkfield - Cholame } \\
2 \mathrm{E}\end{array}$ & 6 & 4.08 & 522.74 & 0.48 & 0.51 & 18.92 & 22.43 & 1.645 & 2.43 \\
\hline 27 & $\begin{array}{l}\text { Parkfield-02, } \\
\text { CA }\end{array}$ & $\begin{array}{c}\text { Parkfield - Cholame } \\
\text { 3E }\end{array}$ & 6 & 5.55 & 397.36 & 0.52 & 0.8 & 22.57 & 27.70 & 1.98 & 2.15 \\
\hline 28 & $\begin{array}{l}\text { Parkfield-02, } \\
\text { CA }\end{array}$ & $\begin{array}{c}\text { Parkfield - Cholame } \\
4 \mathrm{~W}\end{array}$ & 6 & 4.23 & 410.4 & 0.58 & 0.51 & 16.47 & 18.41 & 4.285 & 4.525 \\
\hline 29 & $\begin{array}{l}\text { Parkfield-02, } \\
\text { CA }\end{array}$ & $\begin{array}{c}\text { Parkfield - Fault } \\
\text { Zone } 11\end{array}$ & 6 & 4 & 541.73 & 0.6 & 1.13 & 37.59 & 41.42 & 3.725 & 3.57 \\
\hline
\end{tabular}


Table 4. Earthquake ground motions employed for the analyses of SDOF systems (continued)

\begin{tabular}{|c|c|c|c|c|c|c|c|c|c|c|c|}
\hline \multirow{2}{*}{ No. } & \multirow{2}{*}{$\begin{array}{l}\text { Earthquake } \\
\text { Name }\end{array}$} & \multirow{2}{*}{ Station Name } & \multirow{2}{*}{ Mw } & \multirow{2}{*}{$\begin{array}{l}\mathbf{R}_{\text {rup }} \\
(\mathbf{k m})\end{array}$} & \multirow{2}{*}{$\begin{array}{l}\mathbf{V}_{\mathrm{s} 30} \\
(\mathbf{m} / \mathbf{s})\end{array}$} & \multicolumn{2}{|c|}{ PGA (g) } & \multicolumn{2}{|c|}{ PGA/PGV (Hz) } & \multicolumn{2}{|c|}{$\mathbf{T}_{\mathrm{d}}(\mathrm{sec})$} \\
\hline & & & & & & $\mathbf{L N}$ & TR & $\mathbf{L N}$ & TR & $\mathbf{L N}$ & TR \\
\hline 30 & $\begin{array}{c}\text { Parkfield-02, } \\
\text { CA }\end{array}$ & $\begin{array}{c}\text { Parkfield - Gold Hill } \\
\text { 3E }\end{array}$ & 6 & 6.3 & 450.61 & 0.21 & 0.11 & 14.72 & 15.15 & 6.94 & 7.905 \\
\hline 31 & $\begin{array}{l}\text { Parkfield-02, } \\
\text { CA }\end{array}$ & $\begin{array}{c}\text { Parkfield - Gold Hill } \\
3 \mathrm{~W}\end{array}$ & 6 & 5.41 & 510.92 & 0.79 & 0.43 & 33.42 & 26.04 & 1.44 & 2.535 \\
\hline 32 & $\begin{array}{l}\text { Parkfield-02, } \\
\text { CA }\end{array}$ & $\begin{array}{c}\text { Parkfield - Gold Hill } \\
4 \mathrm{~W}\end{array}$ & 6 & 8.27 & 421.2 & 0.41 & 0.36 & 34.10 & 34.61 & 5.55 & 5.675 \\
\hline 33 & $\begin{array}{l}\text { Parkfield-02, } \\
\text { CA }\end{array}$ & $\begin{array}{c}\text { Parkfield - Stone } \\
\text { Corral 2E } \\
\end{array}$ & 6 & 5.8 & 566.33 & 0.18 & 0.19 & 14.62 & 18.99 & 8.115 & 8.195 \\
\hline 34 & $\begin{array}{l}\text { Parkfield-02, } \\
\text { CA }\end{array}$ & $\begin{array}{c}\text { Parkfield - Stone } \\
\text { Corral 3E } \\
\end{array}$ & 6 & 8.08 & 565.08 & 0.2 & 0.22 & 22.31 & 20.14 & 6.825 & 7.78 \\
\hline 35 & $\begin{array}{c}\text { Parkfield-02, } \\
\text { CA }\end{array}$ & $\begin{array}{c}\text { Parkfield - Vineyard } \\
\text { Cany 1E }\end{array}$ & 6 & 2.96 & 381.27 & 0.27 & 0.29 & 8.50 & 11.00 & 5.1 & 4.07 \\
\hline 36 & $\begin{array}{l}\text { Parkfield-02, } \\
\text { CA }\end{array}$ & $\begin{array}{c}\text { Parkfield - Vineyard } \\
\text { Cany 2E }\end{array}$ & 6 & 4.46 & 467.76 & 0.37 & 0.23 & 13.31 & 17.55 & 4.145 & 5.115 \\
\hline 37 & $\begin{array}{c}\text { Parkfield-02, } \\
\text { CA }\end{array}$ & $\begin{array}{c}\text { Parkfield - Vineyard } \\
\text { Cany } 2 W\end{array}$ & 6 & 3.52 & 438.74 & 0.54 & 0.38 & 17.36 & 18.81 & 3.03 & 4.425 \\
\hline 38 & $\begin{array}{c}\text { Parkfield-02, } \\
\text { CA }\end{array}$ & $\begin{array}{c}\text { Parkfield - Vineyard } \\
\text { Cany } 4 \mathrm{~W}\end{array}$ & 6 & 7.32 & 386.19 & 0.1 & 0.09 & 12.92 & 12.48 & 4.715 & 5.1 \\
\hline 39 & $\begin{array}{c}\text { Parkfield-02, } \\
\text { CA }\end{array}$ & $\begin{array}{c}\text { PARKFIELD - } \\
\text { UPSAR } 02 \\
\end{array}$ & 6 & 9.95 & 416.82 & 0.31 & 0.17 & 17.89 & 11.51 & 7.095 & 9.39 \\
\hline 40 & $\begin{array}{c}\text { Parkfield-02, } \\
\text { CA }\end{array}$ & $\begin{array}{l}\text { PARKFIELD - } \\
\text { UPSAR } 03\end{array}$ & 6 & 9.95 & 440.59 & 0.25 & 0.15 & 15.57 & 13.22 & 9.8 & 11.32 \\
\hline 41 & $\begin{array}{l}\text { Parkfield-02, } \\
\text { CA }\end{array}$ & $\begin{array}{l}\text { PARKFIELD - } \\
\text { UPSAR } 05\end{array}$ & 6 & 9.61 & 440.59 & 0.37 & 0.24 & 17.13 & 13.11 & 10.385 & 11.525 \\
\hline 42 & $\begin{array}{l}\text { Parkfield-02, } \\
\text { CA }\end{array}$ & $\begin{array}{l}\text { PARKFIELD - } \\
\text { UPSAR } 06\end{array}$ & 6 & 9.61 & 440.59 & 0.25 & 0.23 & 13.24 & 16.98 & 11.175 & 13.15 \\
\hline 43 & $\begin{array}{l}\text { Parkfield-02, } \\
\text { CA }\end{array}$ & $\begin{array}{l}\text { PARKFIELD - } \\
\text { UPSAR } 07\end{array}$ & 6 & 9.61 & 440.59 & 0.33 & 0.38 & 17.02 & 22.35 & 10.675 & 11.055 \\
\hline 44 & $\begin{array}{l}\text { Parkfield-02, } \\
\text { CA }\end{array}$ & $\begin{array}{l}\text { PARKFIELD - } \\
\text { UPSAR } 08\end{array}$ & 6 & 9.41 & 440.59 & 0.26 & 0.18 & 16.86 & 14.25 & 10.99 & 12.74 \\
\hline 45 & $\begin{array}{l}\text { Parkfield-02, } \\
\text { CA }\end{array}$ & $\begin{array}{l}\text { PARKFIELD - } \\
\text { UPSAR } 09\end{array}$ & 6 & 9.34 & 466.12 & 0.28 & 0.2 & 12.40 & 9.73 & 11.245 & 11.39 \\
\hline 46 & $\begin{array}{l}\text { Parkfield-02, } \\
\text { CA }\end{array}$ & $\begin{array}{l}\text { PARKFIELD - } \\
\text { UPSAR } 11\end{array}$ & 6 & 9.41 & 466.12 & 0.47 & 0.36 & 17.86 & 17.98 & 9.725 & 9.155 \\
\hline 47 & $\begin{array}{l}\text { Parkfield-02, } \\
\text { CA }\end{array}$ & $\begin{array}{l}\text { PARKFIELD - } \\
\text { UPSAR } 11\end{array}$ & 6 & 9.41 & 466.12 & 0.47 & 0.36 & 17.86 & 17.98 & 9.725 & 9.155 \\
\hline 48 & $\begin{array}{l}\text { Parkfield-02, } \\
\text { CA }\end{array}$ & $\begin{array}{l}\text { PARKFIELD - } \\
\text { UPSAR } 12\end{array}$ & 6 & 9.47 & 466.12 & 0.23 & 0.25 & 17.69 & 11.09 & 9.255 & 10.74 \\
\hline 49 & $\begin{array}{l}\text { Parkfield-02, } \\
\text { CA }\end{array}$ & $\begin{array}{l}\text { PARKFIELD - } \\
\text { UPSAR } 13\end{array}$ & 6 & 9.47 & 466.12 & 0.3 & 0.23 & 17.54 & 11.90 & 12.815 & 11.77 \\
\hline 50 & $\begin{array}{l}\text { Duzce, } \\
\text { Turkey }\end{array}$ & IRIGM 487 & 7.14 & 2.65 & 690 & 0.28 & 0.3 & 8.72 & 7.16 & 12.976 & 14.496 \\
\hline 51 & $\begin{array}{l}\text { Duzce, } \\
\text { Turkey }\end{array}$ & IRIGM 498 & 7.14 & 3.58 & 425 & 0.35 & 0.4 & 14.99 & 11.69 & 11.868 & 12.004 \\
\hline 52 & $\begin{array}{c}\text { Parkfield-02, } \\
\text { CA }\end{array}$ & Hog Canyon & 6 & 5.28 & 376 & 0.28 & 0.26 & 12.13 & 12.72 & 8.93 & 10.08 \\
\hline 53 & $\begin{array}{c}\text { Irpinia, Italy- } \\
01 \\
\end{array}$ & Auletta & 6.9 & 9.55 & 476.62 & 0.06 & 0.06 & 15.12 & 8.38 & 14.9698 & 14.5667 \\
\hline 54 & $\begin{array}{c}\text { Irpinia, Italy- } \\
01 \\
\end{array}$ & Bagnoli Irpinio & 6.9 & 8.18 & 649.67 & 0.13 & 0.19 & 4.22 & 5.69 & 11.4637 & 8.6681 \\
\hline 55 & $\begin{array}{c}\text { Irpinia, Italy- } \\
02\end{array}$ & Calitri & 6.2 & 8.83 & 455.93 & 0.15 & 0.18 & 5.37 & 5.79 & 13.2624 & 12.996 \\
\hline 56 & $\begin{array}{l}\text { Kalamata, } \\
\text { Greece-01 }\end{array}$ & Kalamata (bsmt) & 6.2 & 6.45 & 382.21 & 0.24 & 0.27 & 6.85 & 11.03 & 3.8808 & 3.2352 \\
\hline 57 & $\begin{array}{c}\text { Umbria } \\
\text { Marche, Italy }\end{array}$ & Nocera Umbra & 6 & 8.92 & 428 & 0.47 & 0.38 & 15.48 & 14.20 & 3.575 & 3.715 \\
\hline 58 & $\begin{array}{l}\text { L'Aquila, } \\
\text { Italy }\end{array}$ & $\begin{array}{c}\text { GRAN SASSO } \\
\text { (Assergi) }\end{array}$ & 6.3 & 6.4 & 488 & 0.15 & 0.14 & 13.16 & 17.20 & 7.36 & 7.31 \\
\hline 59 & $\begin{array}{l}\text { L'Aquila, } \\
\text { Italy }\end{array}$ & $\begin{array}{l}\text { L'Aquila - V. Aterno } \\
\text { - Centro Valle }\end{array}$ & 6.3 & 6.27 & 475 & 0.66 & 0.56 & 14.70 & 12.88 & 6.875 & 6.615 \\
\hline 60 & $\begin{array}{l}\text { L'Aquila, } \\
\text { Italy }\end{array}$ & $\begin{array}{c}\text { L'Aquila - V. Aterno } \\
\text {-Colle Grilli }\end{array}$ & 6.3 & 6.81 & 685 & 0.48 & 0.52 & 13.34 & 14.70 & 7.355 & 7.39 \\
\hline 61 & $\begin{array}{l}\text { L'Aquila, } \\
\text { Italy }\end{array}$ & $\begin{array}{c}\text { L'Aquila - V. Aterno } \\
\text {-F. Aterno }\end{array}$ & 6.3 & 6.55 & 552 & 0.4 & 0.44 & 12.17 & 15.78 & 6.92 & 6.59 \\
\hline 62 & $\begin{array}{l}\text { L'Aquila, } \\
\text { Italy }\end{array}$ & L'Aquila - Parking & 6.3 & 5.38 & 717 & 0.34 & 0.36 & 10.49 & 9.54 & 7.995 & 8.39 \\
\hline
\end{tabular}


Table 4. Earthquake ground motions employed for the analyses of SDOF systems (continued)

\begin{tabular}{|c|c|c|c|c|c|c|c|c|c|c|c|}
\hline \multirow{2}{*}{ No. } & \multirow{2}{*}{$\begin{array}{l}\text { Earthquake } \\
\text { Name }\end{array}$} & \multirow{2}{*}{ Station Name } & \multirow{2}{*}{ Mw } & \multirow{2}{*}{$\begin{array}{l}\mathbf{R}_{\text {rup }} \\
(\mathbf{k m})\end{array}$} & \multirow{2}{*}{$\begin{array}{l}\mathbf{V}_{\mathrm{s} 30} \\
(\mathbf{m} / \mathbf{s})\end{array}$} & \multicolumn{2}{|c|}{ PGA (g) } & \multicolumn{2}{|c|}{ PGA/PGV (Hz) } & \multicolumn{2}{|c|}{$\mathbf{T}_{\mathrm{d}}(\mathrm{sec})$} \\
\hline & & & & & & $\mathbf{L N}$ & TR & $\mathbf{L N}$ & TR & $\mathbf{L N}$ & TR \\
\hline 58 & $\begin{array}{l}\text { L'Aquila, } \\
\text { Italy }\end{array}$ & $\begin{array}{c}\text { GRAN SASSO } \\
\text { (Assergi) }\end{array}$ & 6.3 & 6.4 & 488 & 0.15 & 0.14 & 13.16 & 17.20 & 7.36 & 7.31 \\
\hline 59 & $\begin{array}{l}\text { L'Aquila, } \\
\text { Italy }\end{array}$ & $\begin{array}{l}\text { L'Aquila - V. Aterno } \\
\text { - Centro Valle }\end{array}$ & 6.3 & 6.27 & 475 & 0.66 & 0.56 & 14.70 & 12.88 & 6.875 & 6.615 \\
\hline 60 & $\begin{array}{l}\text { L'Aquila, } \\
\text { Italy }\end{array}$ & $\begin{array}{l}\text { L'Aquila - V. Aterno } \\
\text {-Colle Grilli }\end{array}$ & 6.3 & 6.81 & 685 & 0.48 & 0.52 & 13.34 & 14.70 & 7.355 & 7.39 \\
\hline 61 & $\begin{array}{l}\text { L'Aquila, } \\
\text { Italy }\end{array}$ & $\begin{array}{c}\text { L'Aquila - V. Aterno } \\
\text {-F. Aterno }\end{array}$ & 6.3 & 6.55 & 552 & 0.4 & 0.44 & 12.17 & 15.78 & 6.92 & 6.59 \\
\hline 62 & $\begin{array}{l}\text { L'Aquila, } \\
\text { Italy }\end{array}$ & L'Aquila - Parking & 6.3 & 5.38 & 717 & 0.34 & 0.36 & 10.49 & 9.54 & 7.995 & 8.39 \\
\hline 63 & $\begin{array}{c}\text { Nahanni, } \\
\text { Canada }\end{array}$ & Site 1 & 6.76 & 9.6 & 605.04 & 1.11 & 1.2 & 19.92 & 23.65 & 6.835 & 7.085 \\
\hline 64 & $\begin{array}{c}\text { Nahanni, } \\
\text { Canada }\end{array}$ & Site 2 & 6.76 & 4.93 & 605.04 & 0.52 & 0.36 & 14.99 & 10.19 & 6.5975 & 7.04 \\
\hline 65 & $\begin{array}{c}\text { Nahanni, } \\
\text { Canada }\end{array}$ & Site 3 & 6.76 & 5.32 & 605.04 & 0.18 & 0.17 & 31.13 & 55.75 & 5.9725 & 5.92 \\
\hline 66 & $\begin{array}{c}\text { Cape } \\
\text { Mendocino }\end{array}$ & Cape Mendocino & 7.01 & 6.96 & 567.78 & 1.49 & 1.04 & 11.95 & 24.05 & 4.74 & 4.96 \\
\hline 67 & $\begin{array}{c}\text { Cape } \\
\text { Mendocino }\end{array}$ & Petrolia & 7.01 & 8.18 & 422.17 & 0.59 & 0.66 & 11.73 & 7.31 & 17.34 & 15.88 \\
\hline 68 & Northridge-01 & $\begin{array}{c}\text { Jensen Filter Plant } \\
\text { Generator Building }\end{array}$ & 6.69 & 5.43 & 525.79 & 0.57 & 0.99 & 5.48 & 13.96 & 5.335 & 5.215 \\
\hline 69 & Northridge-01 & $\begin{array}{c}\text { LA - Sepulveda VA } \\
\text { Hospital }\end{array}$ & 6.69 & 8.44 & 380.06 & 0.75 & 0.93 & 8.55 & 10.33 & 6.87 & 6.135 \\
\hline 70 & Northridge-01 & LA Dam & 6.69 & 5.92 & 628.99 & 0.43 & 0.32 & 4.87 & 4.50 & 6.395 & 6.2 \\
\hline $71^{*}$ & Northridge-01 & $\begin{array}{c}\begin{array}{c}\text { Pacoima Kagel } \\
\text { Canyon }\end{array} \\
\end{array}$ & 6.69 & 7.26 & 508.08 & 0.3 & 0.43 & 7.86 & 7.80 & 10.1 & 9.84 \\
\hline $72^{*}$ & Northridge-01 & $\begin{array}{c}\text { Sylmar - Olive View } \\
\text { Med FF } \\
\end{array}$ & 6.69 & 5.3 & 440.54 & 0.6 & 0.84 & 7.59 & 6.37 & 5.74 & 4.94 \\
\hline 73 & $\begin{array}{l}\text { Chi-Chi, } \\
\text { Taiwan-03 }\end{array}$ & TCU078 & 6.2 & 7.62 & 443.04 & 0.45 & 0.27 & 7.69 & 13.43 & 5.02 & 5.16 \\
\hline 74 & $\begin{array}{l}\text { Chi-Chi, } \\
\text { Taiwan-03 }\end{array}$ & TCU084 & 6.2 & 9.32 & 665.2 & 0.14 & 0.07 & 7.22 & 3.70 & 14.075 & 14.305 \\
\hline 75 & $\begin{array}{l}\text { Chi-Chi, } \\
\text { Taiwan-03 }\end{array}$ & TCU089 & 6.2 & 9.81 & 671.52 & 0.09 & 0.09 & 7.92 & 10.80 & 6.29 & 5.38 \\
\hline 76 & $\begin{array}{c}\text { San Simeon, } \\
\text { CA }\end{array}$ & $\begin{array}{c}\text { Templeton - 1-story } \\
\text { Hospital }\end{array}$ & 6.5 & 6.22 & 410.66 & 0.44 & 0.48 & 10.07 & 19.91 & 7.62 & 7.155 \\
\hline $77^{*}$ & Niigata, Japan & NIG028 & 6.63 & 9.79 & 430.71 & 0.52 & 0.85 & 12.75 & 26.88 & 5.09 & 4.515 \\
\hline 78 & Niigata, Japan & NIGH01 & 6.63 & 9.46 & 480.4 & 0.67 & 0.84 & 10.19 & 11.77 & 6.515 & 6.325 \\
\hline $79^{*}$ & Niigata, Japan & NIGH11 & 6.63 & 8.93 & 375 & 0.6 & 0.46 & 9.38 & 12.69 & 5.255 & 7.14 \\
\hline 80 & $\begin{array}{l}\text { Montenegro, } \\
\text { Yugo. }\end{array}$ & $\begin{array}{l}\text { Bar-Skupstina } \\
\text { Opstine }\end{array}$ & 7.1 & 6.98 & 462.23 & 0.37 & 0.37 & 8.64 & 6.92 & 16.15 & 16.11 \\
\hline 81 & $\begin{array}{c}\text { Montenegro, } \\
\text { Yugo. }\end{array}$ & $\begin{array}{c}\text { Petrovac - Hotel } \\
\text { Olivia }\end{array}$ & 7.1 & 8.01 & 543.26 & 0.46 & 0.3 & 11.78 & 10.73 & 9.46 & 9.51 \\
\hline 82 & $\begin{array}{l}\text { Montenegro, } \\
\text { Yugo. }\end{array}$ & $\begin{array}{c}\text { Ulcinj - Hotel } \\
\text { Albatros }\end{array}$ & 7.1 & 4.35 & 410.35 & 0.18 & 0.23 & 8.83 & 7.58 & 10.16 & 10.16 \\
\hline 83 & Iwate & IWTH24 & 6.9 & 5.18 & 486.41 & 0.44 & 0.52 & 15.68 & 14.24 & 14.58 & 20.83 \\
\hline $84^{*}$ & Iwate & IWTH25 & 6.9 & 4.8 & 506.44 & 1.43 & 1.15 & 21.84 & 14.93 & 10 & 11.55 \\
\hline 85 & Iwate & $\begin{array}{c}\text { Mizusawaku Interior } \\
\text { O ganecho }\end{array}$ & 6.9 & 7.85 & 413.04 & 0.26 & 0.36 & 13.05 & 10.65 & 24.24 & 31.09 \\
\hline $86^{*}$ & $\begin{array}{l}\text { N. Palm } \\
\text { Springs }\end{array}$ & Cabazon & 6.06 & 7.92 & 376.91 & 0.22 & 0.2 & 27.76 & 14.28 & 4.615 & 4.35 \\
\hline 87 & $\begin{array}{l}\text { N. Palm } \\
\text { Springs }\end{array}$ & $\begin{array}{c}\text { Whitewater Trout } \\
\text { Farm }\end{array}$ & 6.06 & 6.04 & 425.02 & 0.48 & 0.63 & 12.25 & 20.10 & 3.08 & 2.83 \\
\hline $88^{*}$ & Loma Prieta & Corralitos & 6.93 & 3.85 & 462.24 & 0.64 & 0.48 & 10.67 & 8.65 & 5.415 & 5.895 \\
\hline $89^{*}$ & Loma Prieta & $\begin{array}{l}\text { Gilroy - Gavilan } \\
\text { Coll. }\end{array}$ & 6.93 & 9.96 & 729.65 & 0.36 & 0.33 & 10.19 & 12.25 & 2.845 & 2.73 \\
\hline $90^{*}$ & Loma Prieta & LGPC & 6.93 & 3.88 & 594.83 & 0.57 & 0.61 & 5.64 & 9.94 & 5.82 & 6.41 \\
\hline 91 & Loma Prieta & $\begin{array}{c}\text { Saratoga - Aloha } \\
\text { Ave }\end{array}$ & 6.93 & 8.5 & 380.89 & 0.51 & 0.33 & 12.51 & 5.62 & 6.3 & 6.2 \\
\hline
\end{tabular}


Table 4. Earthquake ground motions employed for the analyses of SDOF systems (continued)

\begin{tabular}{|c|c|c|c|c|c|c|c|c|c|c|c|}
\hline \multirow{2}{*}{ No. } & \multirow{2}{*}{$\begin{array}{c}\text { Earthquake } \\
\text { Name }\end{array}$} & \multirow{2}{*}{ Station Name } & \multirow{2}{*}{ Mw } & \multirow{2}{*}{$\begin{array}{l}\mathbf{R}_{\text {rup }} \\
(\mathbf{k m})\end{array}$} & \multirow{2}{*}{$\begin{array}{l}\mathbf{V}_{\mathrm{s} 30} \\
(\mathbf{m} / \mathbf{s})\end{array}$} & \multicolumn{2}{|c|}{ PGA (g) } & \multicolumn{2}{|c|}{ PGA/PGV (Hz) } & \multicolumn{2}{|c|}{$\mathbf{T}_{\mathrm{d}}(\mathrm{sec})$} \\
\hline & & & & & & $\mathbf{L N}$ & TR & $\mathbf{L N}$ & TR & $\mathbf{L N}$ & TR \\
\hline 92 & $\begin{array}{l}\text { Chi-Chi, } \\
\text { Taiwan }\end{array}$ & CHY006 & 7.62 & 9.76 & 438.19 & 0.36 & 0.36 & 8.50 & 5.92 & 17.884 & 18.04 \\
\hline $93^{*}$ & $\begin{array}{l}\text { Chi-Chi, } \\
\text { Taiwan }\end{array}$ & СHY024 & 7.62 & 9.62 & 427.73 & 0.28 & 0.17 & 5.00 & 4.38 & 20.115 & 20.99 \\
\hline $94^{*}$ & $\begin{array}{l}\text { Chi-Chi, } \\
\text { Taiwan }\end{array}$ & CHY028 & 7.62 & 3.12 & 542.61 & 0.64 & 0.76 & 8.92 & 7.90 & 6.015 & 5.055 \\
\hline $95^{*}$ & $\begin{array}{l}\text { Chi-Chi, } \\
\text { Taiwan }\end{array}$ & CHY080 & 7.62 & 2.69 & 496.21 & 0.81 & 0.86 & 7.81 & 9.10 & 8.125 & 13.185 \\
\hline $96^{*}$ & $\begin{array}{l}\text { Chi-Chi, } \\
\text { Taiwan } \\
\end{array}$ & TCU049 & 7.62 & 3.76 & 487.27 & 0.28 & 0.24 & 4.64 & 4.25 & 19.125 & 20.805 \\
\hline $97^{*}$ & $\begin{array}{l}\text { Chi-Chi, } \\
\text { Taiwan } \\
\end{array}$ & TCU050 & 7.62 & 9.49 & 542.41 & 0.15 & 0.13 & 4.16 & 2.65 & 22.53 & 23.34 \\
\hline $98^{*}$ & $\begin{array}{l}\text { Chi-Chi, } \\
\text { Taiwan } \\
\end{array}$ & TCU052 & 7.62 & 0.66 & 579.1 & 0.36 & 0.45 & 2.04 & 2.05 & 14.12 & 14.91 \\
\hline $99^{*}$ & $\begin{array}{l}\text { Chi-Chi, } \\
\text { Taiwan } \\
\end{array}$ & TCU053 & 7.62 & 5.95 & 454.55 & 0.23 & 0.13 & 5.43 & 2.70 & 20.355 & 24.485 \\
\hline $100^{*}$ & $\begin{array}{l}\text { Chi-Chi, } \\
\text { Taiwan } \\
\end{array}$ & TCU054 & 7.62 & 5.28 & 460.69 & 0.15 & 0.19 & 3.15 & 4.52 & 20.685 & 24.485 \\
\hline $101^{*}$ & $\begin{array}{l}\text { Chi-Chi, } \\
\text { Taiwan } \\
\end{array}$ & TCU060 & 7.62 & 8.51 & 375.42 & 0.2 & 0.1 & 5.81 & 2.33 & 19.395 & 19.895 \\
\hline $102^{*}$ & $\begin{array}{l}\text { Chi-Chi, } \\
\text { Taiwan }\end{array}$ & TCU063 & 7.62 & 9.78 & 476.14 & 0.18 & 0.13 & 3.86 & 1.49 & 26.46 & 26.14 \\
\hline 103 & $\begin{array}{l}\text { Chi-Chi, } \\
\text { Taiwan }\end{array}$ & TCU068 & 7.62 & 0.32 & 487.34 & 0.51 & 0.37 & 1.73 & 1.21 & 9.19 & 12.58 \\
\hline 104 & $\begin{array}{l}\text { Chi-Chi, } \\
\text { Taiwan }\end{array}$ & TCU071 & 7.62 & 5.8 & 624.85 & 0.53 & 0.65 & 9.33 & 10.09 & 20.945 & 19.86 \\
\hline 105 & $\begin{array}{l}\text { Chi-Chi, } \\
\text { Taiwan }\end{array}$ & TCU072 & 7.62 & 7.08 & 468.14 & 0.48 & 0.38 & 6.34 & 7.18 & 20.565 & 22.3 \\
\hline 106 & $\begin{array}{c}\text { Chi-Chi, } \\
\text { Taiwan }\end{array}$ & TCU075 & 7.62 & 0.89 & 573.02 & 0.33 & 0.26 & 2.81 & 6.78 & 24.94 & 25.39 \\
\hline 107 & $\begin{array}{l}\text { Chi-Chi, } \\
\text { Taiwan }\end{array}$ & TCU076 & 7.62 & 2.74 & 614.98 & 0.34 & 0.43 & 5.65 & 6.91 & 24.515 & 24.445 \\
\hline 108 & $\begin{array}{l}\text { Chi-Chi, } \\
\text { Taiwan }\end{array}$ & TCU078 & 7.62 & 8.2 & 443.04 & 0.45 & 0.31 & 11.46 & 9.27 & 22.73 & 24.855 \\
\hline 109 & $\begin{array}{l}\text { Chi-Chi, } \\
\text { Taiwan }\end{array}$ & TCU082 & 7.62 & 5.16 & 472.81 & 0.23 & 0.19 & 4.28 & 4.60 & 20.44 & 23.86 \\
\hline 110 & $\begin{array}{l}\text { Chi-Chi, } \\
\text { Taiwan }\end{array}$ & TCU087 & 7.62 & 6.98 & 538.69 & 0.12 & 0.11 & 2.61 & 2.94 & 19.12 & 19.69 \\
\hline 111 & $\begin{array}{l}\text { Chi-Chi, } \\
\text { Taiwan }\end{array}$ & TCU089 & 7.62 & 9 & 671.52 & 0.35 & 0.23 & 8.50 & 6.10 & 23.675 & 22.665 \\
\hline 112 & $\begin{array}{l}\text { Chi-Chi, } \\
\text { Taiwan } \\
\end{array}$ & TCU101 & 7.62 & 2.11 & 389.41 & 0.21 & 0.26 & 2.86 & 5.70 & 16.045 & 16.22 \\
\hline 113 & $\begin{array}{l}\text { Chi-Chi, } \\
\text { Taiwan } \\
\end{array}$ & TCU102 & 7.62 & 1.49 & 714.27 & 0.3 & 0.17 & 3.08 & 2.12 & 13.615 & 15.43 \\
\hline 114 & $\begin{array}{l}\text { Chi-Chi, } \\
\text { Taiwan } \\
\end{array}$ & TCU103 & 7.62 & 6.08 & 494.1 & 0.13 & 0.15 & 1.67 & 4.08 & 14.945 & 15.63 \\
\hline 115 & $\begin{array}{l}\text { Chi-Chi, } \\
\text { Taiwan } \\
\end{array}$ & TCU120 & 7.62 & 7.4 & 459.34 & 0.23 & 0.2 & 3.39 & 4.84 & 24.495 & 24.02 \\
\hline 116 & $\begin{array}{l}\text { Chi-Chi, } \\
\text { Taiwan } \\
\end{array}$ & TCU122 & 7.62 & 9.34 & 475.46 & 0.21 & 0.26 & 3.82 & 5.07 & 23 & 20.58 \\
\hline 117 & $\begin{array}{l}\text { Chi-Chi, } \\
\text { Taiwan }\end{array}$ & TCU129 & 7.62 & 1.83 & 511.18 & 1 & 0.62 & 14.73 & 11.43 & 23.405 & 24.935 \\
\hline 118 & $\begin{array}{l}\text { Chi-Chi, } \\
\text { Taiwan }\end{array}$ & TCU136 & 7.62 & 8.27 & 462.1 & 0.17 & 0.17 & 3.21 & 3.01 & 16.432 & 16.816 \\
\hline 119 & $\begin{array}{l}\text { Chi-Chi, } \\
\text { Taiwan }\end{array}$ & TCU138 & 7.62 & 9.78 & 652.85 & 0.21 & 0.21 & 4.57 & 4.64 & 28.24 & 27.928 \\
\hline 120 & $\begin{array}{l}\text { Christchurch, } \\
\text { New Zealand }\end{array}$ & $\begin{array}{l}\text { Heathcote Valley } \\
\text { Primary School }\end{array}$ & 6.2 & 3.36 & 422 & 1.65 & 1.29 & 16.23 & 20.82 & 4.335 & 4.96 \\
\hline 121 & $\begin{array}{l}\text { Christchurch, } \\
\text { New Zealand }\end{array}$ & LPCC & 6.2 & 6.12 & 649.67 & 0.91 & 0.95 & 22.25 & 23.64 & 3.82 & 3.505 \\
\hline $122^{*}$ & $\begin{array}{l}\text { Mammoth } \\
\text { Lakes-01 }\end{array}$ & Convict Creek & 6.06 & 6.63 & 382.12 & 0.42 & 0.44 & 17.34 & 18.34 & 8.69 & 8.925 \\
\hline
\end{tabular}




\section{Analysis procedure and results}

\subsection{Response of the SDOF systems}

In this section, the implemented methodology and related outcomes are discussed. At first, all of the 244 acceleration time histories were scaled record by record so as their maximum acceleration was equal to $1.0 \mathrm{~g}$, that is, each record was divided by its PGA. The 5-95\% significant duration $\left(T_{d}\right)$ of each time history along with 2.0 seconds of the time history before the $T_{d}$ was considered. Then, a time interval of sufficient length with zero acceleration was inserted at the end of each time history.

It is worth noting that the aforementioned time interval was embedded at the end of the time histories in order to monitor the velocity response of the systems during the free vibration. In fact, when successive ground motions (i.e., mainshock-aftershock seismic sequences) happen, there is often a time gap between the mainshock and the subsequent aftershocks which may be lasting from a few minutes to even a several days. Since the cost of analysis is of a great importance specially in the nonlinear time history analysis, an optimized estimation of the required time gap (i.e., a specified interval of the time with zero acceleration during which the structural model's velocity response approaches a value close to zero or the time needed for the structure to stop free vibration.) is necessary to be determined. For this purpose, a sufficient time gap was applied at the end of the acceleration time histories to make sure that the velocity response of the SDOF systems reaches a value very close to zero $\left(V_{\text {zero }}\right)$. In this study, it is assumed that if the free vibration velocity response of the system becomes less than or equal to the $0.1 \%$ of the maximum free vibration velocity response $\left(V_{\max . f}\right)$ of the system and it does not increase anymore, the structural model stops free vibration (Eq. (2)). In this study, the upper limit $V_{\text {zero }}$ in Eq. (2), was set to $0.1 \%$ of the $V_{\max . f}$.

$$
V_{\text {system }} \leq V_{\text {zero }}
$$

For this purpose, the time required for the free vibrations velocity of the system to reach $V_{\text {zero }}$ was extracted using trial and error procedure. Thus, zero acceleration interval of 50 seconds was initially considered. If the assumed interval is not enough for approaching zero velocity, the interval will be increased to 100 seconds. This would be applied iteratively until the required rest time is extracted with acceptable accuracy.

After performing the dynamic time history analyses on the SDOF systems, the velocity responses were processed and the required time for each SDOF system to stop free vibration (rest time) was extracted according to the method discussed above. It should be noted that the same procedure was done for the MDOF systems supposed that the velocity responses of the roof level be used. Rest time of the SDOF systems were plotted against the 5-95\% significant duration of the ground motions. Fig. 8 shows the resulted plots for the SDOF systems with fundamental period $(\mathrm{T})$ of $1.0 \mathrm{~s}$ and $4.0 \mathrm{~s}$.
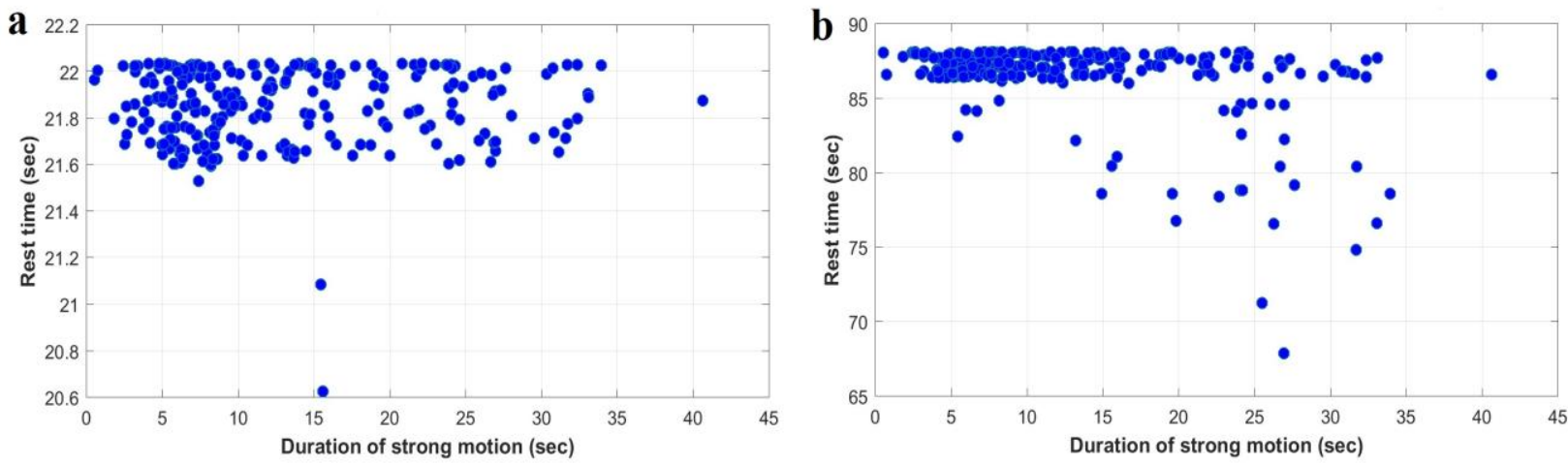

Fig. 8. Evaluated rest time of the SDOF systems versus strong motion duration: (a) SDOF system with fundamental period (T) of $1.0 \mathrm{sec}$., (b) SDOF system with $\mathrm{T}=4.0 \mathrm{sec}$.

In another try, rest time of the SDOF systems were normalized by 5-95\% significant duration of the corresponding record and a comparison was carried out between the normalized rest time data series $\left(\mathrm{R}_{\mathrm{T}}\right)$ and the significant duration $\left(T_{d}\right)$ of the earthquake records to observe the changing trend. The related plots for the SDOF systems with $\mathrm{T}=1.0 \mathrm{~s}$ and $\mathrm{T}=4.0 \mathrm{~s}$ are illustrated in Fig. 9 (a) and Fig. 9 (b) respectively. 

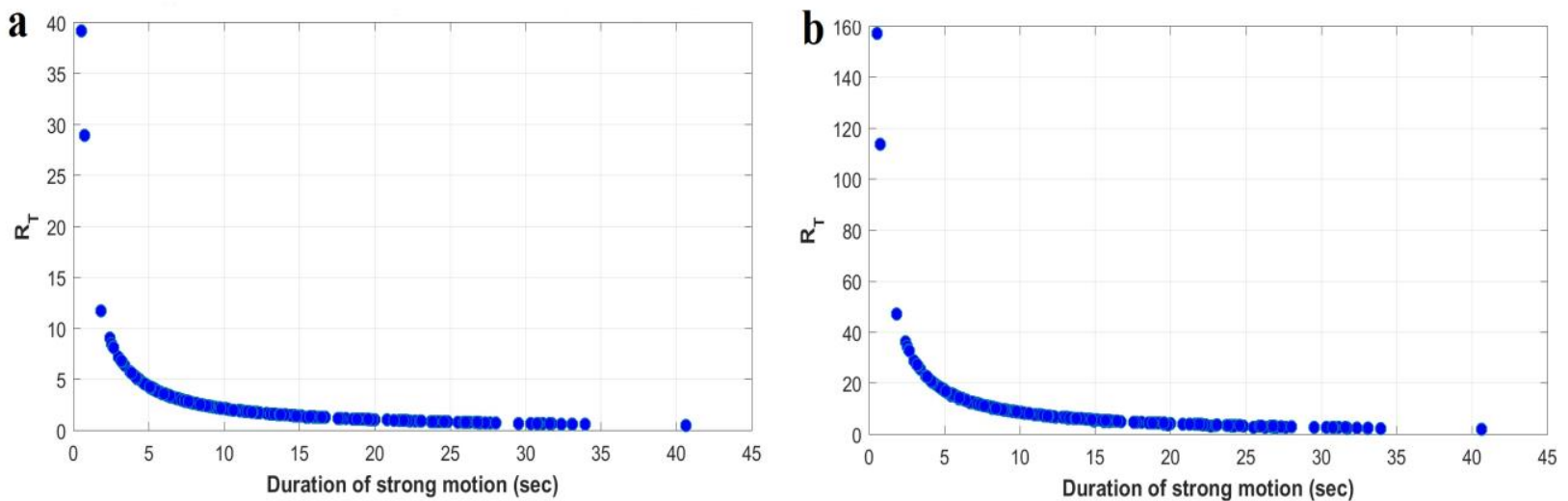

Fig. 9. Normalized rest time $\left(\mathrm{R}_{\mathrm{T}}\right)$ of the SDOF systems versus strong motion duration: (a) SDOF system with $\mathrm{T}=$ 1.0 sec., (b) SDOF system with $\mathrm{T}=4.0 \mathrm{sec}$.

According to the Fig. 9 (a) and Fig. 9 (b), it is obvious that the normalized rest time ( $\left.R_{T}\right)$ of an SDOF system is strongly correlated with the strong motion duration of the applied ground motions and as the strong motion duration increases, the normalized rest time decreases in a regular nonlinear trend, but results of the mere rest time (nonnormalized data), versus strong motion duration (see Fig. 8) looks less encouraging. It seems that the rest time is not straightly related to the strong motion duration as it is depicted in Fig. 8, but by normalizing the evaluated rest time with respect to the corresponding strong motion duration of each earthquake record, the negative nonlinear correlation of the normalized rest time and the strong motion duration data series becomes evident.

The strong emerged relationship between the parameters $\mathrm{R}_{\mathrm{T}}$ and $\mathrm{T}_{\mathrm{d}}$ for the SDOF systems with fundamental period (T) ranging from 0.05 to $7.0 \mathrm{~s}$, leads to curve fitting of data series. This way, evaluated normalized rest time of the SDOF systems $\left(\mathrm{R}_{\mathrm{T}}\right)$ was expressed as the function of strong motion duration $\left(\mathrm{T}_{\mathrm{d}}\right)$ and the fundamental vibration period (T) of the SDOF systems. Details on the computational tool and the mathematical operations on the data series is explained in the following section.

\subsection{Proposed formulation and related mathematical computation}

As mentioned in the earlier section, for each of the SDOF systems with a given vibrational period (T), the normalized rest time $\left(\mathrm{R}_{\mathrm{T}}\right)$ data series were plotted against the strong motion duration $\left(\mathrm{T}_{\mathrm{d}}\right)$ for all the 244 applied earthquake components. Therefore, 22 (number of SDOF systems) graphs illustrating $\mathrm{R}_{\mathrm{T}}$ against $\mathrm{T}_{\mathrm{d}}$ for each SDOF system such as the one in Fig. 9(a) were extracted. The observations showed that the normalized rest time data series $\left(\mathrm{R}_{\mathrm{T}}\right)$ and the strong motion duration data series $\left(\mathrm{T}_{\mathrm{d}}\right)$ are strongly correlated in a nonlinear reverse order in all the cases. Accordingly, regression analyses were performed on each of the outcome graphs so as to find the curve that best fits to the observed data points. The curve fitting technique utilizing the nonlinear least squares method was employed in this study to extract the aforementioned curves or functions (Hansen et al. 2013). The general form of the assumed function for the fitted curve is written as the form of Eq. (3):

$$
y=a x^{b}+c
$$

where $\mathrm{x}$ indicates the strong motion duration of each earthquake $\left(\mathrm{T}_{\mathrm{d}}\right)$, $\mathrm{y}$ is the estimated normalized rest time $\left(\mathrm{R}_{\mathrm{T}}\right.$, estimate) with respect to $T_{d}$ data and $a, b$ and $c$ are the coefficient of the equation which are obtained by fitting the assumed function to the observed data points. Thus the Eq. (3) is converted to the following form of Eq. (4):

$$
R_{\text {T.estimate }}=a\left(T_{d}\right)^{b}+c
$$

By fitting the curve of the assumed form to each of the 22 observed datasets for SDOF systems, the coefficients $\mathrm{a}, \mathrm{b}$ and $\mathrm{c}$ were determined. Examples of the fitted curve versus the observed data (from the time history analyses) are shown in Fig. 10. It can be seen that the fitted curve is matched well by the observed data points. Results for the other examined SDOF systems are similar. 

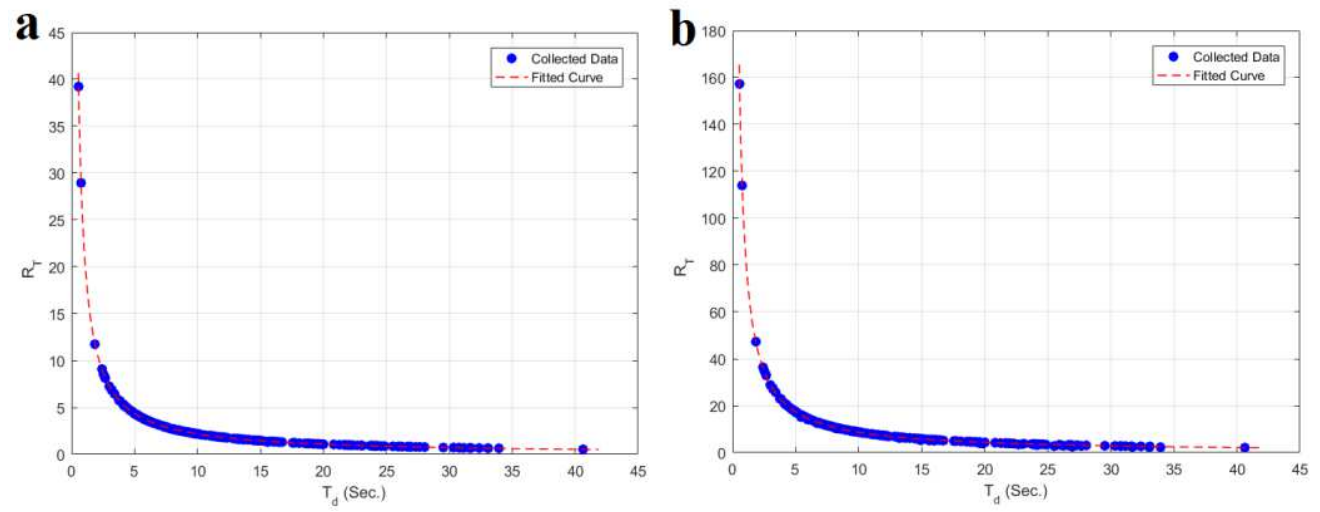

Fig. 10. Curves fitted to the normalized rest time $\left(R_{T}\right)$ plotted versus the strong motion duration $\left(T_{d}\right)$ : $(a)$ for $T=1.0$ $\mathrm{s}$, (b) for $\mathrm{T}=4.0 \mathrm{~s}$

In order to evaluate the goodness of the fit, the percentage of error, defined in Eq. (5) is calculated:

Error $\%=\left(\left(R_{\text {T.estimate }}-R_{T}\right) / R_{T}\right) \times 100$

where $R_{\text {T.estimate }}$ represents the normalized rest time estimated by the fitted function and $R_{T}$ is the normalized required rest time obtained from the SDOF analysis.
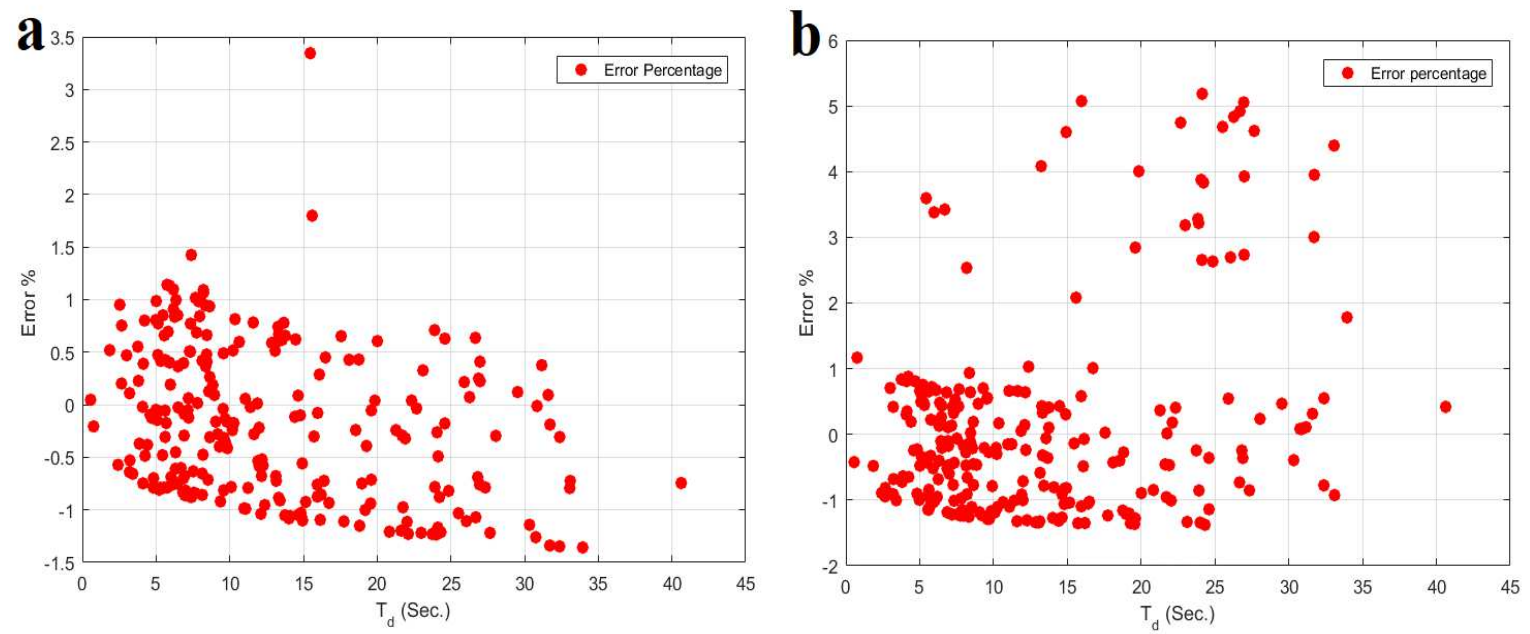

Fig. 11. The percentage of difference between the collected rest time and the determined rest time obtained from the fitted curve's formula: (a) for $\mathrm{T}=1.0 \mathrm{~s}$, (b) for $\mathrm{T}=4.0 \mathrm{~s}$

As shown in Fig. 11, the absolute value of the error percentage is no more than the 5\% meaning that the assumed curve is fitted well enough. Results of the remaining SDOF models are also analogous. Implementation of the curve fitting process resulted in the constant coefficients $\mathrm{a}, \mathrm{b}$ and $\mathrm{c}$ for each of the SDOF systems.

Tracking the changes of the ' $a$ ' coefficient with regard to the changes of the fundamental vibration period of the SDOF systems, a linear relation between the two parameters was figured out as is illustrated in Fig. 12(a). But variation of the two other coefficients ' $b$ ' and ' $c$ ' over the changes of the vibrational period are nearly around zero value as shown in Fig. 12(b) and Fig. 12(c) respectively. Hence, conservatively the upper limit of data was considered for these two coefficients. 

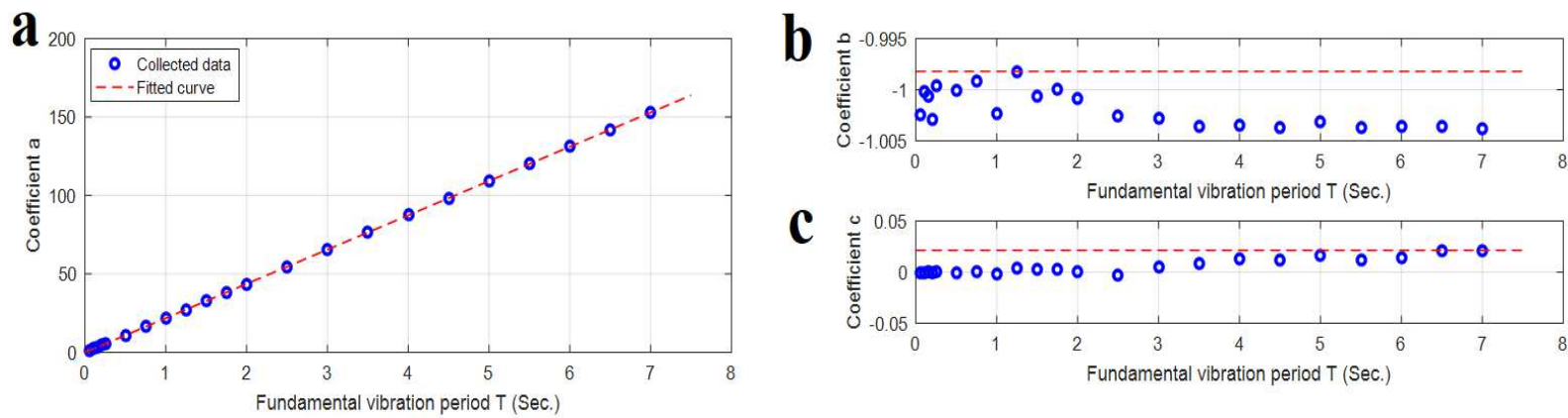

Fig. 12. Curves fitted to the coefficients of the Eq. (3) plotted along with the obtained coefficients as a function of fundamental vibration period of SDOF systems: (a) for "a" coefficient, (b) for "b" coefficient, (c) for "c" coefficient

The coefficients for the SDOF systems with the damping ratio $(\xi)$ of $5 \%$ and the hardening slope (h) equal to $3 \%$ can be expressed as Eq. (6), Eq. (7) and Eq. (8):

$$
\begin{aligned}
& a(T)=21.8559(T)+0.0258 \\
& b=-0.9982 \\
& c=0.0214
\end{aligned}
$$

in which, T represents the fundamental vibration period of the SDOF system and $a(T)$ indicates the 'a' coefficient as a function of the vibration period for the corresponding SDOF system with a specified T. Now, Eq. (4) along with the obtained Eq. $(6,7,8)$ can come together and be written as Eq. (9):

$$
R_{\text {T.estimate }}=(21.8559 T+0.0258)\left(T_{d}\right)^{-0.9982}+0.0214
$$

The proposed equation (Eq. (9)), estimates the optimum normalized rest time of a structure as a function of the vibration period $(\mathrm{T})$ and the strong motion duration of the applied earthquake component $\left(\mathrm{T}_{\mathrm{d}}\right)$. The Estimated normalized rest time $\left(R_{\text {T.estimate }}\right)$, should finally be multiplied by $\mathrm{T}_{\mathrm{d}}$ to obtain the required rest time for the structure (R) as follows:

$$
R=T_{d}\left[(21.8559 T+0.0258)\left(T_{d}\right)^{-0.9982}+0.0214\right]
$$

The proposed formulation estimates the minimum required rest time of a nonlinear system with $\xi=5 \%$ and hardening slope (h) of 3\% which is subjected to the scaled ground motions. In order to develop the use of the proposed formulation in a more general term, influences of the linearity of modeling, ground motion scaling, different hardening slope and the effect of various damping ratios are investigated and the results are explained comprehensively in the following sections.

\subsection{Linearity versus nonlinearity}

In addition to the nonlinear SDOF systems, a group of linear SDOF systems having only the linear part of the bilinear behavior and the same vibrational periods, were also modeled and analyzed. Results of the linear models as well as the nonlinear systems, under scaled ground motions and for the damping ratio of $5 \%$ is illustrated in Fig. 13(a) and Fig. 13(b) for instances.

It is inferred that no matter systems behave linearly or nonlinearly, the required rest time is identical. Therefore, the proposed formulation for the rest time calculation (Eq. (10)), is appropriate for both the linear systems as well as the nonlinear systems. 

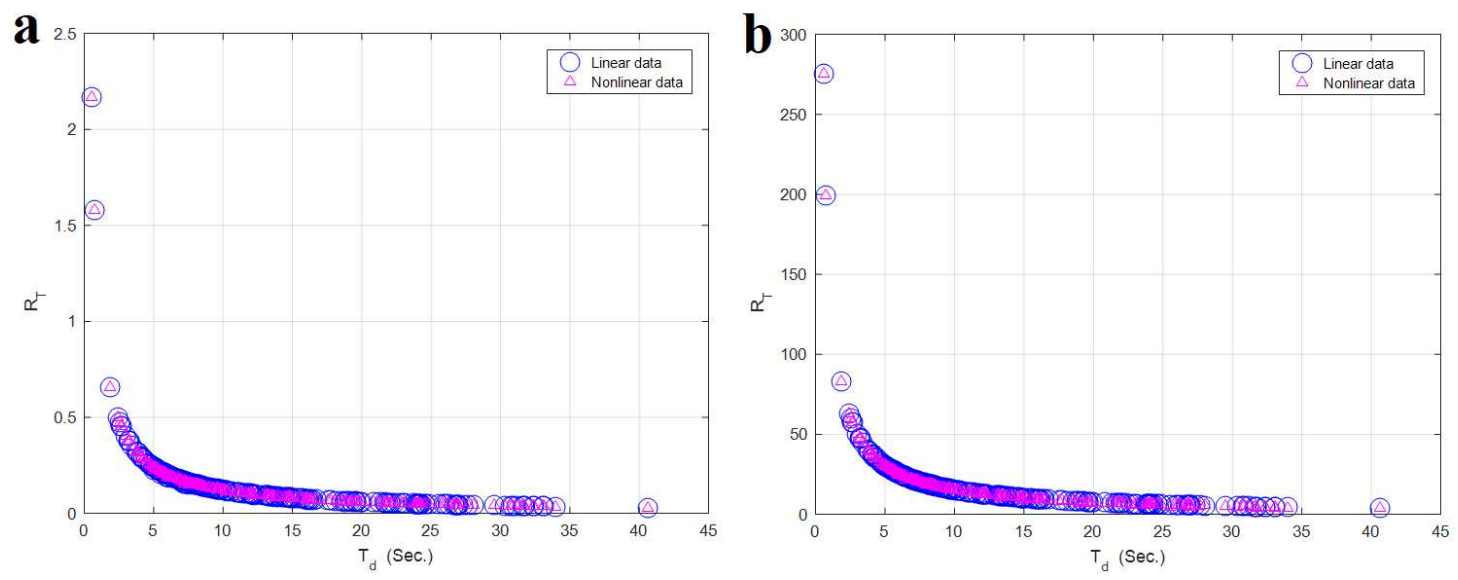

Fig. 13. Normalized rest time $\left(R_{T}\right)$ plotted against strong motion duration $\left(T_{d}\right)$ for the collected data of a linear SDOF system as well as a nonlinear SDOF system: (a) for an SDOF with $\mathrm{T}=0.05 \mathrm{~s}$, (b) for an SDOF with $\mathrm{T}=7.0 \mathrm{~s}$

\subsection{Influence of ground motion scaling}

Ground motion components were once applied as the free field records and in another try, they were scaled to a common value such that their peak ground acceleration (PGA) be equal to 1.0 time the ground acceleration ( $\mathrm{g}$ or $9.806 \mathrm{~m} / \mathrm{s}^{2}$ ) to make a basis for comparison. Rest time results in both the scaled and the free field conditions were extracted separately. A comparison between the normalized rest time of the nonlinear SDOF systems while subjected to the scaled ground motions and the same systems in case they were analyzed under the free field ground motions was conducted.

As is shown in Fig. 14, the effect of ground motion scaling can be ignored since the rest time obtained using scaled ground motion is same as the one yielded by the free field earthquake record. It should be noted that the results shown in Fig. 13 (for nonlinear SDOF systems with 3\% hardening slope and the 5\% damping ratio) were confirmed for the remaining SDOF systems.
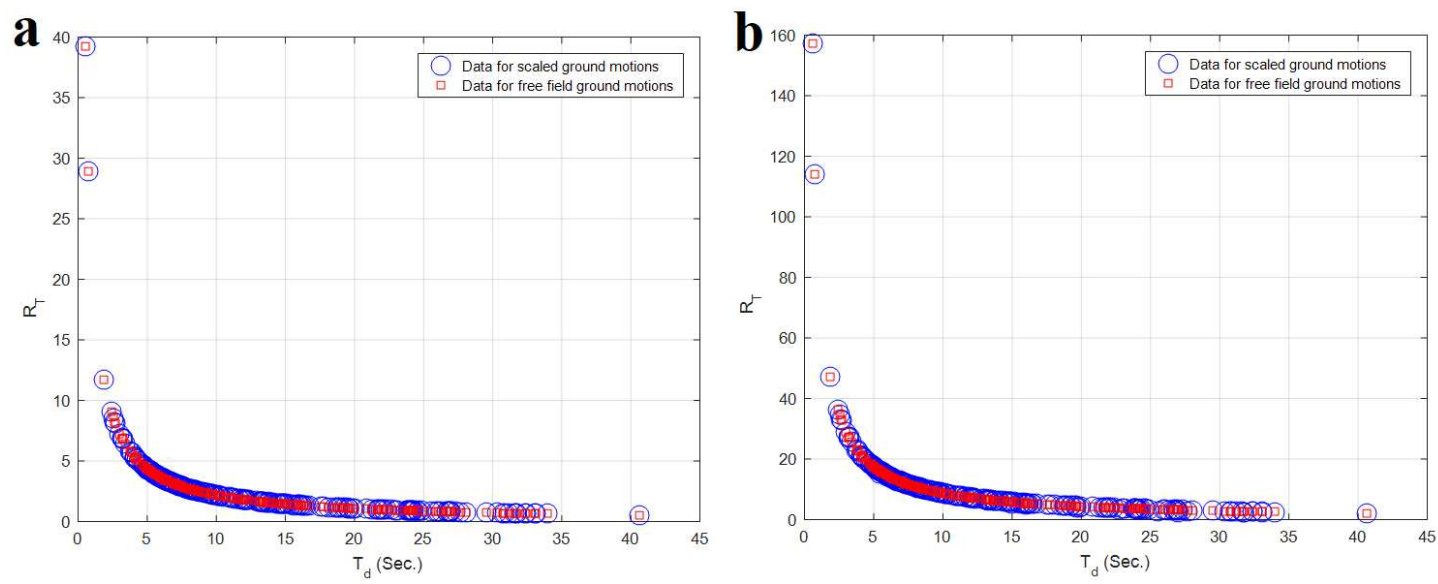

Fig. 14. Normalized rest time $\left(R_{T}\right)$ plotted against strong motion duration $\left(T_{d}\right)$ While using scaled ground motions as well as free field ground motions: (a) for $\mathrm{T}=1.0 \mathrm{~s}$, (b) for $\mathrm{T}=4.0 \mathrm{~s}$

\subsection{Influence of hardening slope}

Impacts of defining different hardening slopes for nonlinear behavior of systems on the required rest time has also been investigated. Observing Fig. 15, it is readily figured out that the rest time outputs for the assumed hardening slope of $3 \%$ conforms to the results for the $1 \%$ hardening slope. Therefore, different assumptions for the hardening slope (h) parameter in the nonlinear description of models, are not going to influence on the required rest time of the system. 

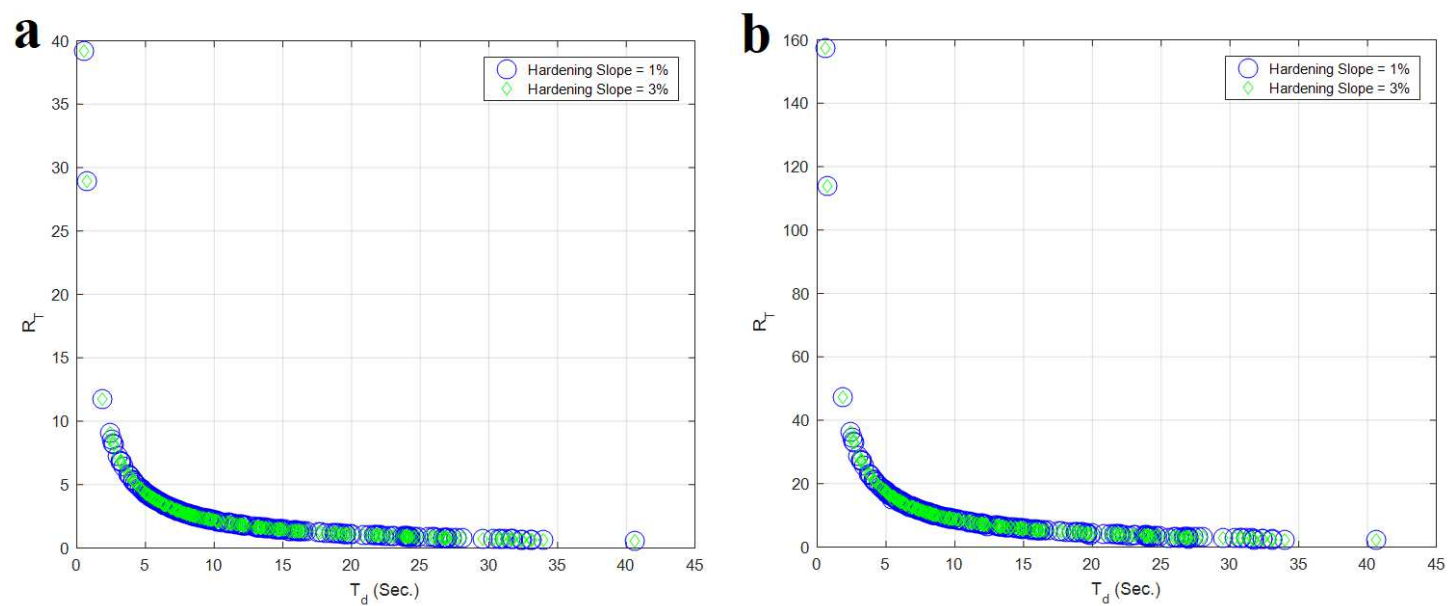

Fig. 15. Normalized rest time $\left(R_{T}\right)$ plotted against the strong motion duration $\left(T_{d}\right)$ for different hardening slopes of nonlinear behavior: (a) for $\mathrm{T}=1.0 \mathrm{~s}$, (b) for $\mathrm{T}=4.0 \mathrm{~s}$

\subsection{Influence of damping ratio}

A key factor that the required rest time of a system is influenced by, is damping ratio since this parameter is directly concerned with the energy dissipation of a system. It is expected as the damping ratio increases, the free vibration of structural system after mainshock be damped more rapidly.

Hence, the effect of above-mentioned parameter was examined for three groups of SDOF systems having damping ratio of $2.5 \%, 5 \%$ and $7.5 \%$ respectively. Rest time results for the three values of damping ratio are presented in Fig. 16. As the damping ratio decreases, the rest time curve moves upward meaning that the rest time demand rises up. Therefore, required rest time of the system is related to the damping ratio conversely.
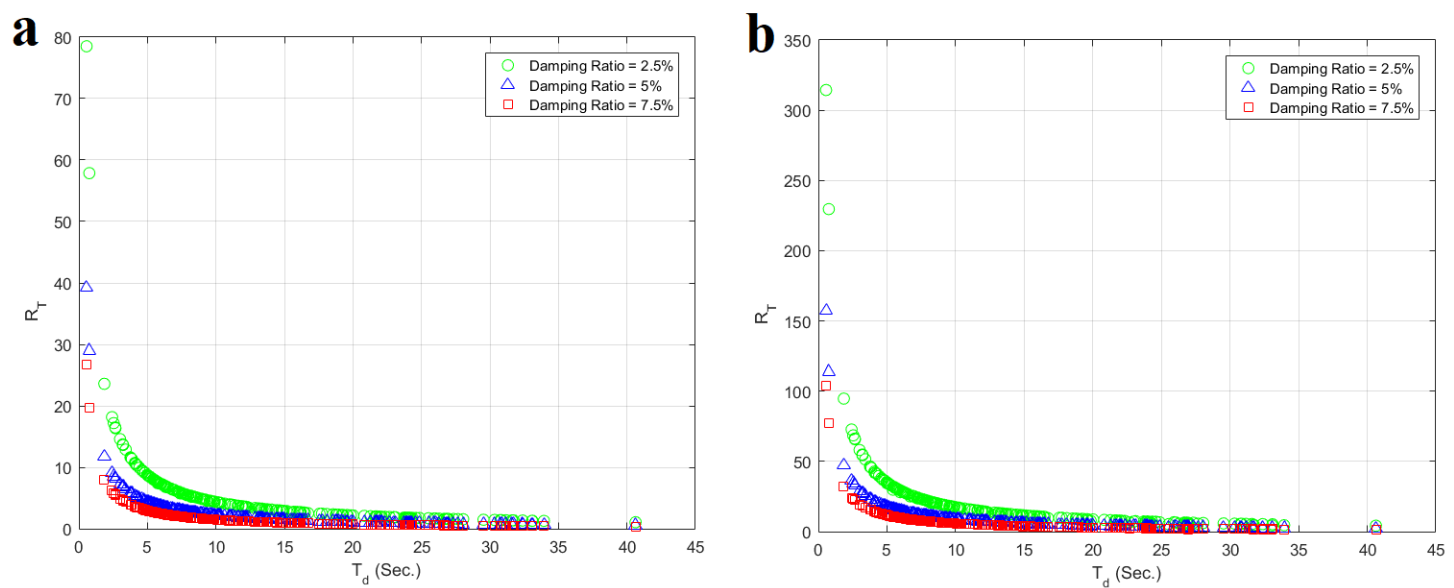

Fig. 16. Normalized rest time $\left(R_{T}\right)$ plotted against the strong motion duration $\left(T_{d}\right)$ data series using different damping ratios: (a) for $\mathrm{T}=1.0 \mathrm{~s}$, (b) for $\mathrm{T}=4.0 \mathrm{~s}$

To deal with the damping ratio effect on the rest time response of the system, ratio of normalized rest time of the SDOF systems while $\xi=2.5 \%\left(R_{T .2 .5 \%}\right)$ to the normalized rest time of the systems while $\xi=5 \%\left(R_{T .5 \%}\right)$, was calculated and compared with $\xi_{5 \%} / \xi_{2.5 \%}$ ratio (which is equal to 0.05/0.025) in Fig. 16 for a SDOF system with $\mathrm{T}=$ $1.0 \mathrm{~s}$ as an example. It is shown that $R_{T .2 .5 \%} / R_{T .5 \%}$ values are almost entirely close to the continuous line of data representing the $\xi_{5 \%} / \xi_{2.5 \%}$ value. For instance, in Fig. 17(a), $\mathrm{R}_{\mathrm{T}}$ ratios vary between 1.95 and 2.05 mostly which means that the $\mathrm{R}_{\mathrm{T}}$ ratios approach the value of $\xi_{5 \%} / \xi_{2.5 \%}$ or 2.0 . 

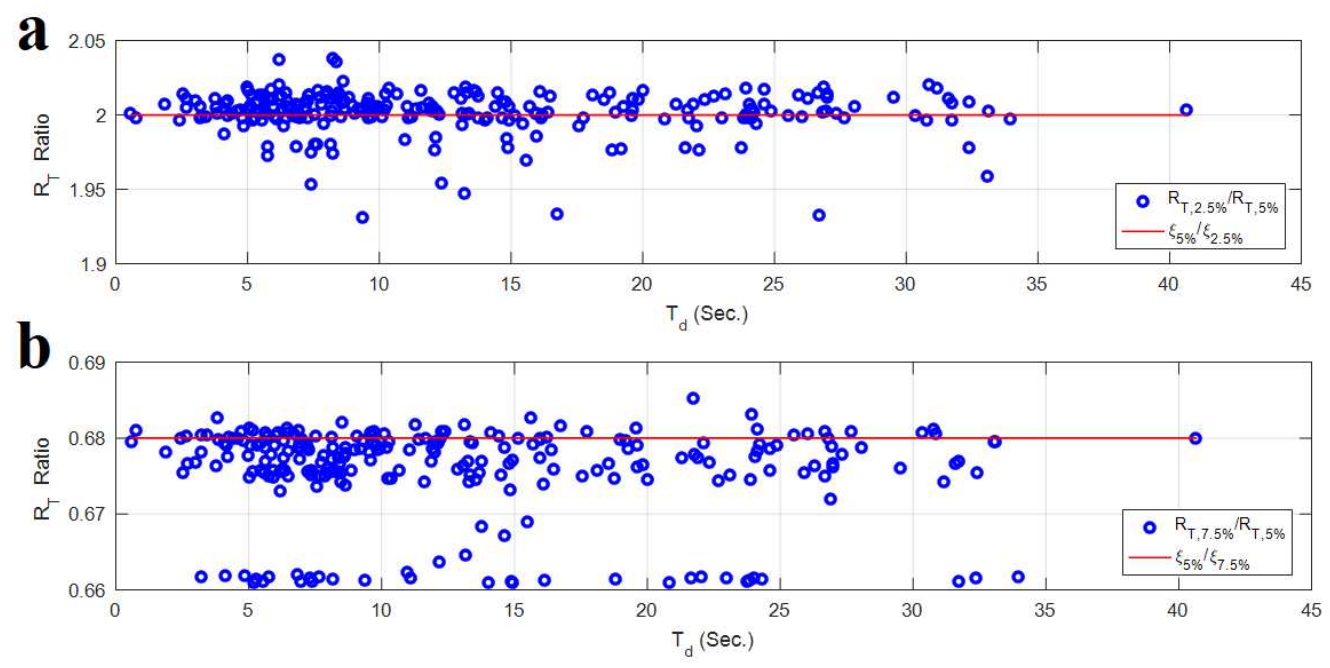

Fig. 17. The ratio of normalized rest time $\left(R_{T}\right)$ versus strong motion duration $\left(T_{d}\right)$ for a SDOF having two different damping ratios with respect to the 5\% damping ratio: (a) for $\xi=2.5 \%$, (b) for $\xi=7.5 \%$

It was demonstrated that for all other examined SDOF systems, the following relation in Eq. (11) is confirmed:

$$
\frac{R_{T . \xi}}{R_{T .5 \%}} \cong \frac{\xi_{5 \%}}{\xi}
$$

in which $R_{T . \xi}$ represents the normalized rest time for a damping ratio of interest $(\xi), R_{T .5 \%}$ is the normalized rest time for $\xi=5 \%$ and the parameters $\xi_{5 \%}$ and $\xi$ are damping ratio of 5\% and target damping, respectively.

The proposed formulation was extracted for the commonly used damping ratio of $5 \%$. In order to generalize the application for any desired damping ratio and take the damping effect into account, Eq. (9), Eq. (10) and Eq. (11) were assembled and resulted in Eq. (12):

$$
R_{\xi}=T_{d}\left(\frac{\xi_{5 \%}}{\xi}\right)\left[(21.8559 T+0.0258)\left(T_{d}\right)^{-0.9982}+0.0214\right]
$$

where $R_{\xi}$ is the required rest time of the system for the arbitrary damping ratio of $\xi$. Therefore, Eq. (10) was modified so that it could deal with the damping ratio effect. Finally, the proposed formulation in Eq. (12) estimates the required rest time of a system as a function of input parameters $T$ or fundamental vibration period of structure, $\xi$ or damping ratio of structure and $T_{d}$ or strong motion duration.

\subsection{Response of MDOF steel frames and validation}

Promising results of the SDOF systems encouraged the authors to evaluate the proposed formulation for the MDOF systems. For this purpose, two steel moment resisting frames with 3 and 12 number of stories representatives of low-rise and mid-rise frames respectively were modeled. Design details and other considerations of the structural models were explained previously in section 3.

Since the ground motions should be commonly scaled in nonlinear analyses, a set of 30 scaled ground motions were applied to each of the steel frames. The ground motions were multiplied by a factor to match the average response spectrum for the $10 \%$ probability of exceedance in 50 years (10/50) hazard level. Also, a damping ratio of $5 \%$ was set for nonlinear dynamic analysis of MDOF systems.

Required rest time for each of the frames were calculated based on the procedure discussed earlier in section 4.1 and were compared to the estimated rest time determined using the proposed formulation in this paper (Eq. (12)). Fig. 18 illustrates the required rest times for each frame versus the estimated rest times extracted from the proposed formulation. 

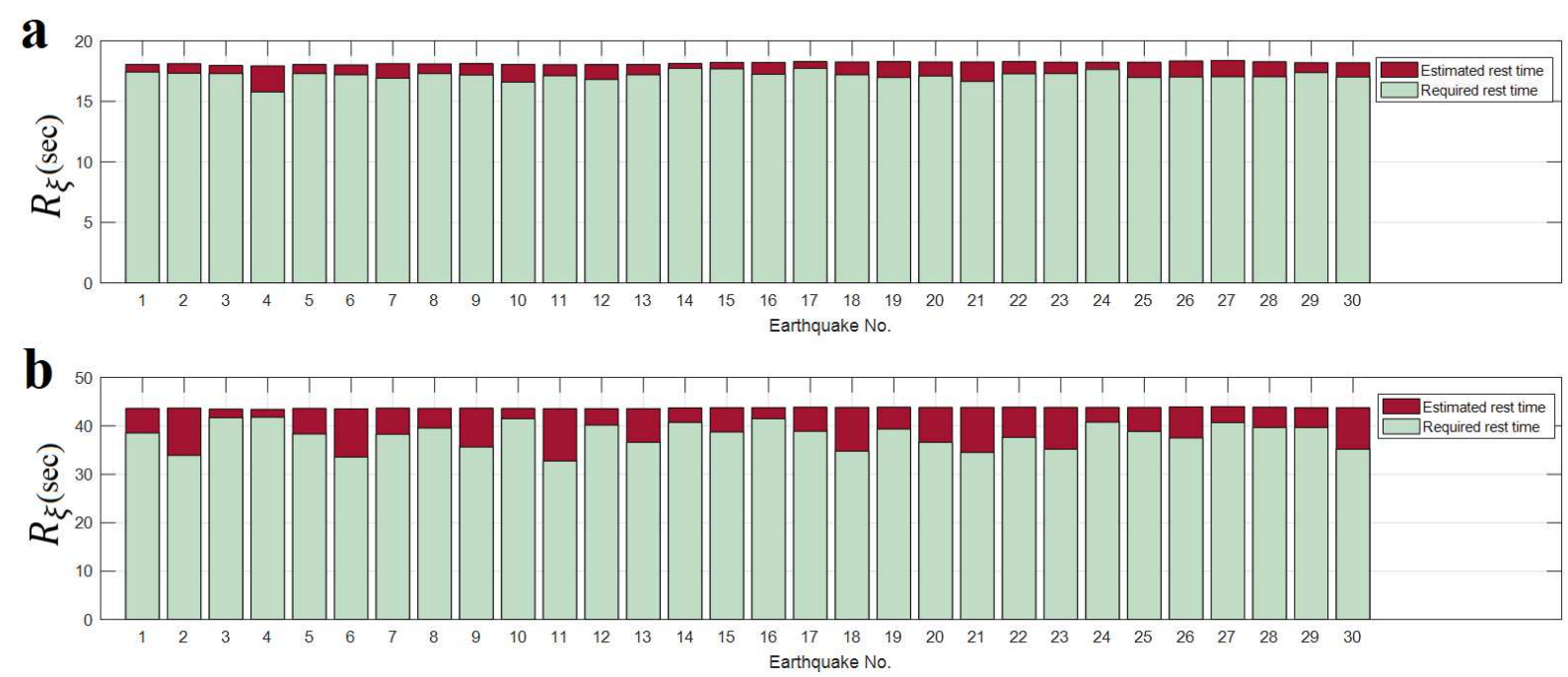

Fig. 18. Required rest time obtained from the analysis versus the estimated rest time using the proposed formulation: (a) for the 3-story steel frame, (b) for the 12-story steel frame

It is observed that the proposed formula gives a good estimation for the required rest time and the differences between the estimated values and the required values are almost small especially for the low-rise frame. In fact, the proposed formula estimates the rest time conservatively.

The agreement between the rest time data obtained from the nonlinear analyses of frames and the rest time data estimated using the proposed formula was also investigated and as the results in Fig. 19 reveal, there is a satisfactory agreement between them. Therefore, the proposed formulation gives an appropriate estimation both for SDOF systems as well as MDOF systems.
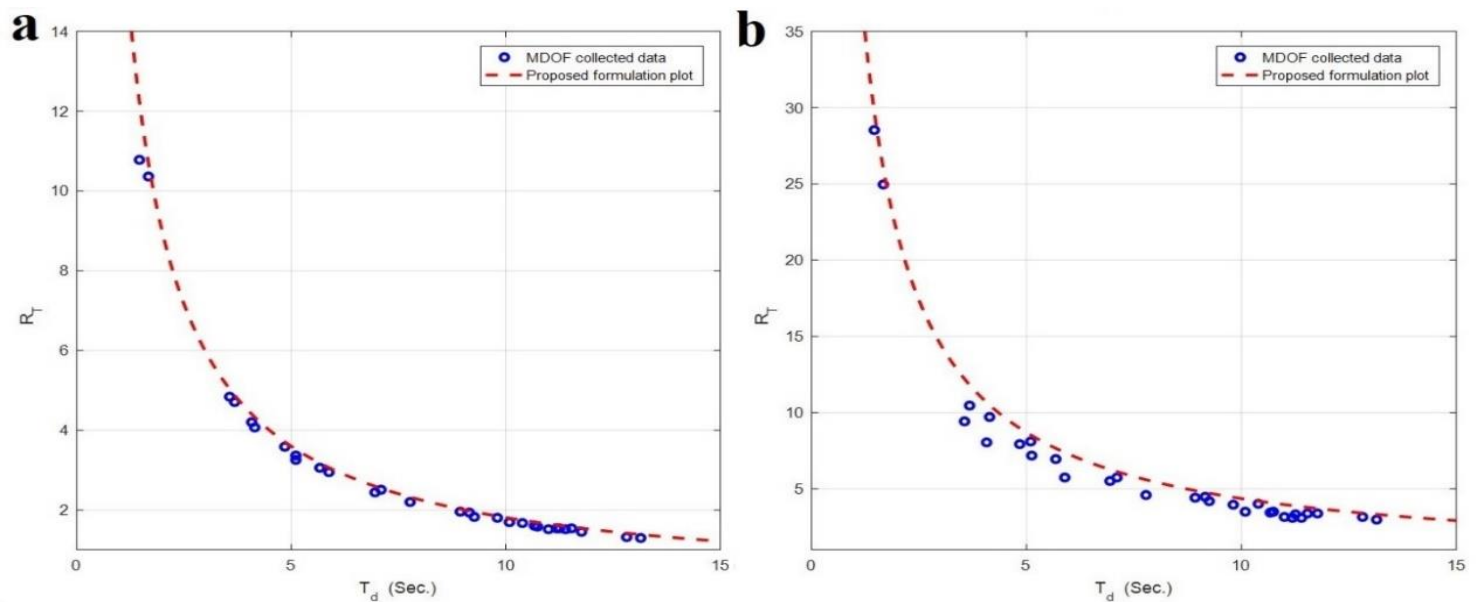

Fig. 19. Normalized rest time $\left(R_{T}\right)$ plotted against the strong motion duration $\left(T_{d}\right)$ data series using different damping ratios: (a) for $\mathrm{T}=1.0 \mathrm{~s}$, (b) for $\mathrm{T}=4.0 \mathrm{~s}$

\section{Discussion}

\subsection{Detailed examination of required rest time against system period and strong motion duration}

To better understand the influence of strong motion duration on the required rest time of structures with different vibration periods, maximum and minimum rest time for each of the vibration periods considering different strong motions (Td), were calculated. Rest time results as a function of vibration periods (T) is displayed in Fig. 20 for the maximum and minimum values. From Fig. 20, first of all it is readily observed that the rest time is totally increased as the system's vibration period is increased.

Fig. 20 also points out to the difference between maximum and minimum required rest time in short periods compared to the long periods. 


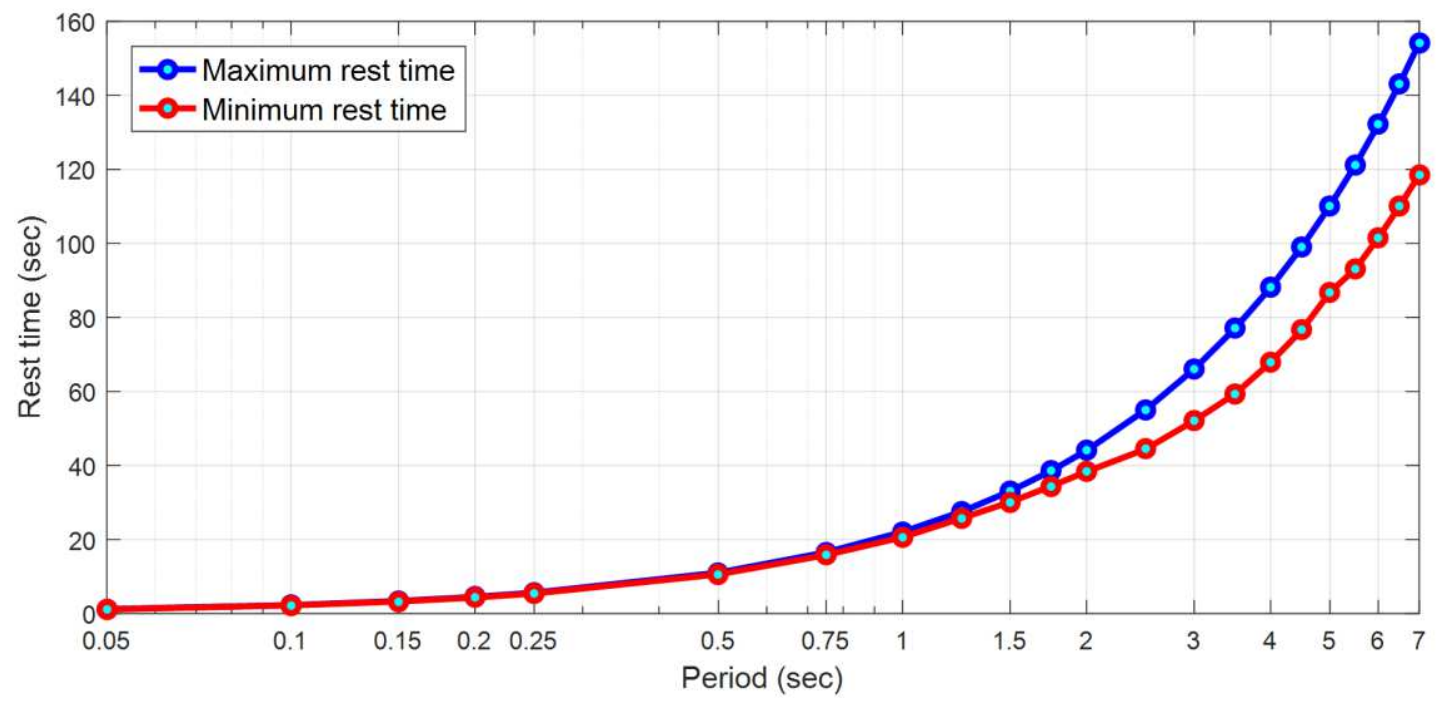

Fig. 20. Maximum and minimum rest time plotted against the vibration period

It is shown that the difference between the maximum and minimum rest times is more apparent in long periods. In fact, for systems with vibration period of less than $1.0 \mathrm{~s}$, the differences are almost negligible. Since the maximum and minimum rest times were calculated considering different strong motion durations, it is inferred that the influence of strong motion duration (Td) on the required rest time of a system with vibrating period of less than $1.0 \mathrm{~s}$ (short periods), is slight and can be ignored but as the period increases (over $1.0 \mathrm{~s}$ ), the impact of strong motion duration appears to be important. For example, for the vibration period of a system being equal to $7.0 \mathrm{~s}$, the difference between the maximum rest time and minimum rest time (which were calculated for two different values of $\mathrm{Td}$ ), is around $20 \mathrm{~s}$ but the difference for a system with vibrating period of $1.0 \mathrm{~s}$ is close to zero value. Therefore, rest time of a system depends strongly on the strong motion duration as well as the vibrating period of system specially for systems with long periods. Fig. 20, also gives a general insight to the required rest time for an applicable range of vibrating periods. It is worth mentioning that the proposed formula conservatively estimates the upper limit value for the rest time.

\subsection{Scope of the study}

It is valuable to clearly explain the limitations and scope of this study. This would greatly help to better understand the main goal of this work. Also, considering these limitations, the future studies to strengthen the findings of this paper can be determined.

As stated earlier, the prime objective of this work is to develop a framework to include the required rest time between mainshock-aftershock sequence in dynamic analysis of structures. The results showed that the time gap between mainshock-aftershock sequence can be efficiently estimated using system properties and strong motion duration of earthquakes. Although these results demonstrate the validity of this claim, but it should be expected that developed formula can evolve if the inputs of described analysis procedure change.

The first important parameter is the selected earthquake records. In this work, relatively a large number of records have been selected, but it cannot be argued that they can represent all seismic characteristic of earthquake ground motion. For example, selected events are all near field records which belong to site class $\mathrm{C}$ according to ASCE site classification. One may expect including mid and far fault events recorded on other type of sites would affect final results.

Second, the effect of stiffness degradation and the strength deterioration was not considered for the SDOF and MDOF models of the structures. This phenomenon may change the required rest time for a structure after the mainshock.

Finally, the evaluated formula by dynamic analysis of SDOF systems has directly applied to MDOF structures. It is worthy to consider structures with different height and lateral resisting frame to improve the formula for real world applications. 
These are the main limitations of this study which determine the scope of presented work. These limitations can be the subject of future studies to finally develop a comprehensive method for estimating required rest time for different structures located on different seismogenetically active regions.

\section{Conclusions}

In this paper, a novel formulation is introduced to estimate the required rest time between mainshock and aftershock in nonlinear dynamic analysis of structures. The proposed formula evaluates the required rest time with respect to structural system's properties (vibrational period) and the ground motion's feature (strong motion duration). A set of 244 ground motion components for the nonlinear dynamic analyses of SDOF systems were considered in the current study. 30 earthquake records were also used for the analyses of steel frames and validation of the obtained results. In order to generalize the proposed formulation, influences of different parameters such as structural behavior (linearity or nonlinearity), ground motion scaling, hardening slope in bilinear behavior and damping ratio were investigated and taken into account. Finally, the proposed formulation was validated for low and mid-rise MDOF steel frames. Important conclusions accomplished in this study are outlined:

- Required rest time is strongly correlated with strong motion duration and vibration period of the structure. Ther efore, the output of the proposed formulation is the required rest time as a function of these two parameters.

- Rest time results for linear models was analogous to the rest time results of the nonlinear models. Hence, the req uired rest time of a system does not depend on the level of nonlinearity that a system experiences.

- Whether using the scaled earthquake ground motions or the free field ones, the required rest time is identical. $\mathrm{T}$ herefore, the effect of ground motion scaling on the required rest time is ignored.

- Application of different hardening slopes in the bilinear modeling of structural behavior demonstrated that the $r$ equired rest time of a structure is not affected by this parameter.

- Investigation of the damping ratio impact on the required rest time has shown that as the damping ratio decrease $\mathrm{s}$, rest time demand increases. The ratio of rest time demand of a system with arbitrary damping ratio to the rest time of a system with 5\% damping is proportioned to the ratio their damping percentage inversely. Therefore, th e proposed formula was modified so that it is applicable for a system with an arbitrary damping ratio.

- There is a good agreement between the required rest obtained from the proposed formula and the required rest ti me computed using the nonlinear analyses of the steel frames. So, the proposed formula can be confidently used to estimate the required rest time of a MDOF system.

Finally, it should be noted that the main purpose of this study (to develop a method for estimating the required rest time) has been met according to the results obtained. However, it can be expected that the proposed formula will be more improved by including the limitations of this work in future studies.

\section{Declarations}

Ethical statements (The developed method is the original effort of the authors which is not submitted or published elsewhere)

Funding (This research was supported by the Kharazmi University Grant. The authors would like to thank the Kharazmi University)

Conflicts of interest/Competing interests (The authors declare that they have no conflict of interest)

Availability of data and material (Data and material are available)

Code availability (The developed codes are available)

Authors' contributions (Conceptualization: [Roohollah Mohammadi Pirooz, Soheila Habashi, Ali Massumi];

Formal Analysis: [Roohollah Mohammadi Pirooz, Soheila Habashi]; Methodology: [Roohollah Mohammadi Pirooz, Soheila Habashi, Ali Massumi]; Validation: [Ali Massumi]; Writing - original draft: [Soheila Habashi]; Writing review \& editing: [Ali Massumi])

Plant Reproducibility (Not applicable)

Clinical Trials Registration (Not applicable)

Gels and Blots/ Image Manipulation (Not applicable)

High Risk Content (Not applicable)

\section{References}

Amadio C, Fragiacomo M, Rajgelj S (2003) The effects of repeated earthquake ground motions on the non-linear 
response of SDOF systems. Earthq Eng Struct Dyn 32:291-308. https://doi.org/10.1002/eqe.225

Chopra AK (2006) Dynamics of structures. theory and applications to, 3rd edn. Prentice Hall Inc., New Jersey

Clough RW, Penzien J (1995) Dynamics of Structures, 3rd edn. Computers \& Structures, Inc., Berkeley

Deierlein G, Baker J, Chandramohan R, Foschaar J (2012) Effect of Long Duration Ground Motions on Structural Performance, PEER Annual Meeting

Far H (2017) Advanced computation methods for soil-structure interaction analysis of structures resting on soft soils. Int J Geotech Eng 13:352-359. https://doi.org/10.1080/19386362.2017.1354510

Goda K (2012a) Peak Ductility Demand of Mainshock-Aftershock Sequences for Japanese Earthquakes. In: Proceedings of the Fifteenth World Conference on Earthquake Engineering, Lisbon, Portugal

Goda K (2012b) Nonlinear response potential of Mainshock-Aftershock sequences from Japanese earthquakes. Bull Seismol Soc Am 102:2139-2156. https://doi.org/10.1785/0120110329

Goda K (2015) Record selection for aftershock incremental dynamic analysis. Earthq Eng Struct Dyn 44:11571162. https://doi.org/10.1002/eqe.2513

Han R, Li Y, van de Lindt J (2015) Assessment of Seismic Performance of Buildings with Incorporation of Aftershocks. J Perform Constr Facil 29:04014088. https://doi.org/10.1061/(ASCE)CF.1943-5509.0000596

Hansen PC, Pereyra V, Scherer G (2013) Least squares data fitting with applications. JHU Press

Hatzigeorgiou GD (2010a) Behavior factors for nonlinear structures subjected to multiple near-fault earthquakes. Comput Struct 88:309-321. https://doi.org/10.1016/j.compstruc.2009.11.006

Hatzigeorgiou GD (2010b) Ductility demand spectra for multiple near- and far-fault earthquakes. Soil Dyn Earthq Eng 30:170-183. https://doi.org/10.1016/j.soildyn.2009.10.003

Hatzigeorgiou GD, Beskos DE (2009) Inelastic displacement ratios for SDOF structures subjected to repeated earthquakes. Eng Struct 31:2744-2755. https://doi.org/10.1016/j.engstruct.2009.07.002

Hatzigeorgiou GD, Liolios AA (2010) Nonlinear behaviour of RC frames under repeated strong ground motions. Soil Dyn Earthq Eng 30:1010-1025. https://doi.org/10.1016/j.soildyn.2010.04.013

Hosseini SA, Ruiz-García J, Massumi A (2019) Seismic response of RC frames under far-field mainshock and nearfault aftershock sequences. Struct Eng Mech 72:395-408. https://doi.org/10.12989/sem.2019.72.3.395

Hoveidae N, Radpour S (2020) Performance evaluation of buckling-restrained braced frames under repeated earthquakes. Bull Earthq Eng 1-22. https://doi.org/10.1007/s10518-020-00983-0

Huang W, Qian J, Fu Q (2012) Damage assessment of RC frame structures under mainshockaftershock seismic sequences. In: Proc. of the 15th World Conference on Earthquake Engineering (15WCEE), Portugal, Lisbon

Li Q, Ellingwood BR (2007) Performance evaluation and damage assessment of steel frame buildings under main shock-aftershock earthquake sequences. Earthq Eng Struct Dyn 36:405-427. https://doi.org/10.1002/eqe.667

Massumi A, Mohammadi R (2016) Structural redundancy of 3D RC frames under seismic excitations. Struct Eng Mech 59:15-36. https://doi.org/10.12989/sem.2016.59.1.015

Massumi A, Monavari B (2013) Energy based procedure to obtain target displacement of reinforced concrete structures. Struct Eng Mech 48:681-695. https://doi.org/10.12989/sem.2013.48.5.681

Mirtaherf M, Amini M, Rad MD (2017) The effect of mainshock-aftershock on the residual displacement of buildings equipped with cylindrical frictional damper. Earthq Struct 12:515-527. https://doi.org/10.12989/eas.2017.12.5.515

Moustafa A, Takewaki I (2011) Response of nonlinear single-degree-of-freedom structures to random acceleration sequences. Eng Struct 33:1251-1258. https://doi.org/10.1016/j.engstruct.2011.01.002

P. Gallagher R, A. Reasenberg P, D. Poland C (1996) The ATC TechBrief 2, Earthquake Aftershocks --Entering Damaged Buildings

Papaloizou L, Polycarpou P, Komodromos P, et al (2016) Two-dimensional numerical investigation of the effects of multiple sequential earthquake excitations on ancient multidrum columns. Earthq Struct 10:495-521. https://doi.org/10.12989/eas.2016.10.3.495

$\mathrm{Pu}$ W, Wu M (2018) Ductility demands and residual displacements of pinching hysteretic timber structures subjected to seismic sequences. Soil Dyn Earthq Eng 114:392-403. https://doi.org/10.1016/j.soildyn.2018.07.037

Ruiz-García J, Negrete-Manriquez JC (2011) Evaluation of drift demands in existing steel frames under as-recorded far-field and near-fault mainshock-aftershock seismic sequences. Eng Struct 33:621-634. https://doi.org/10.1016/j.engstruct.2010.11.021

Samimifar M, Massumi A, Moghadam AS (2019) A new practical equivalent linear model for estimating seismic hysteretic energy demand of bilinear systems. Struct Eng Mech 70:289-301.

https://doi.org/10.12989/sem.2019.70.3.289

Tabatabaiefar S, Fatahi B, Samali B (2012) Finite difference modelling of soil-structure interaction for seismic 
design of moment resisting building frames

Xie X, Lin G, Duan YF, et al (2012) Seismic damage of long span steel tower suspension bridge considering strong aftershocks. Earthq Struct 3:767-781. https://doi.org/10.12989/eas.2012.3.5.767

Zhai CH, Wen WP, Chen ZQ, et al (2013) Damage spectra for the mainshock-aftershock sequence-type ground motions. Soil Dyn Earthq Eng 45:1-12. https://doi.org/10.1016/j.soildyn.2012.10.001

Zhai CH, Wen WP, Li S, et al (2014) The damage investigation of inelastic SDOF structure under the mainshockaftershock sequence-type ground motions. Soil Dyn Earthq Eng 59:30-41. https://doi.org/10.1016/j.soildyn.2014.01.003

Zhang Y, Shen J, Chen J (2020) Damage-based yield point spectra for sequence-type ground motions. Bull Earthq Eng 18:4705-4724. https://doi.org/10.1007/s10518-020-00874-4

Zhu TJ, Tso WK, Heidebrecht AC (1988) Effect of peak ground a/v ratio on structural damage. J Struct Eng 114:1019-1037. https://doi.org/https://doi.org/10.1061/(ASCE)0733-9445(1988)114:5(1019)

(2016) Seismic provisions for structural steel buildings. American Institute of Steel Construction (AISC), Chicago

(2017) Minimum design loads and associated criteria for buildings and other structures - ASCE Standard. American Society of Civil Engineers, Reston 
Figures

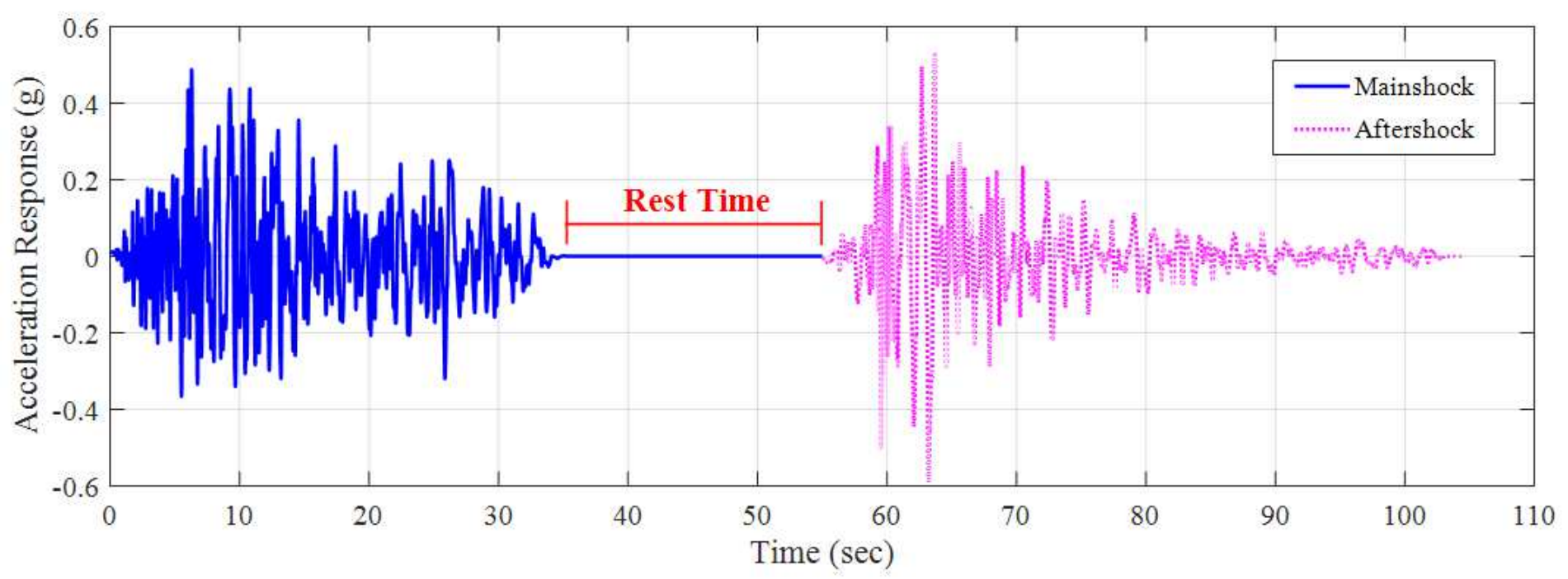

\section{Figure 1}

An illustration of the rest time definition in a mainshock-aftershock earthquake record

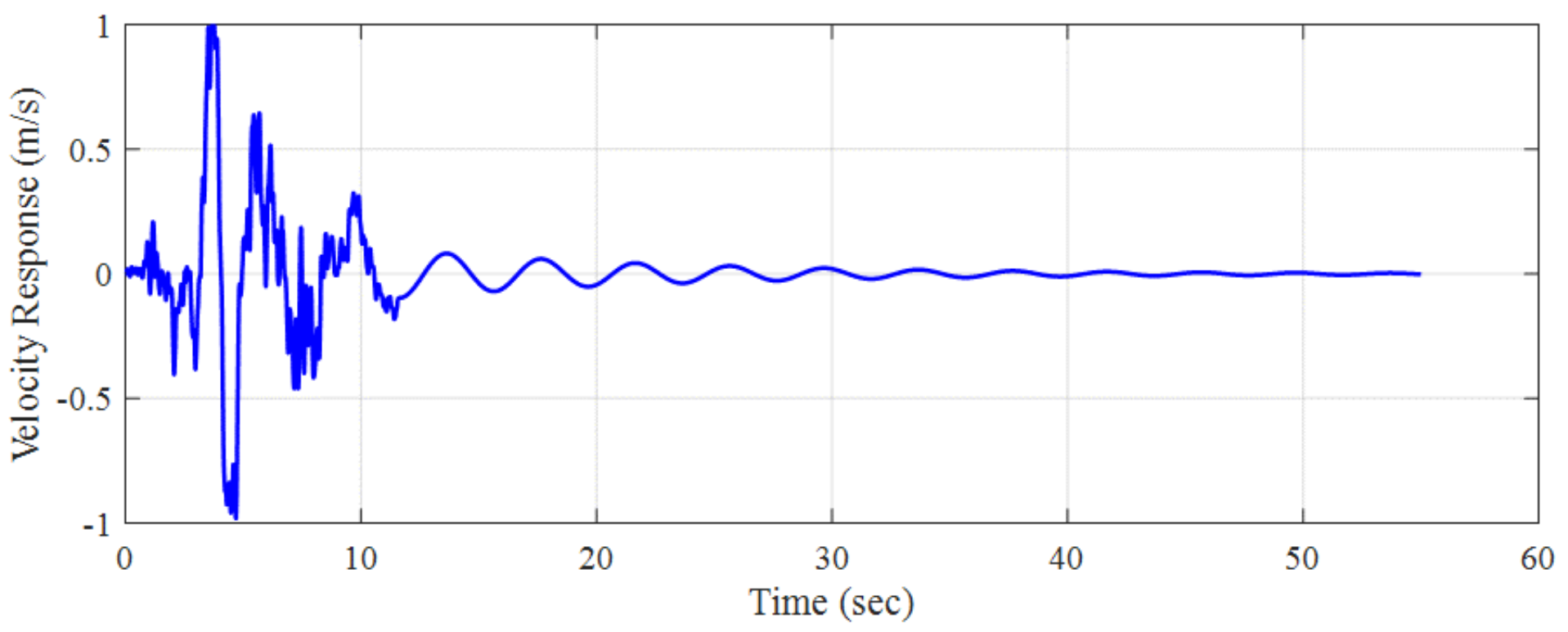

Figure 2

General form of the velocity response of a SDOF system 


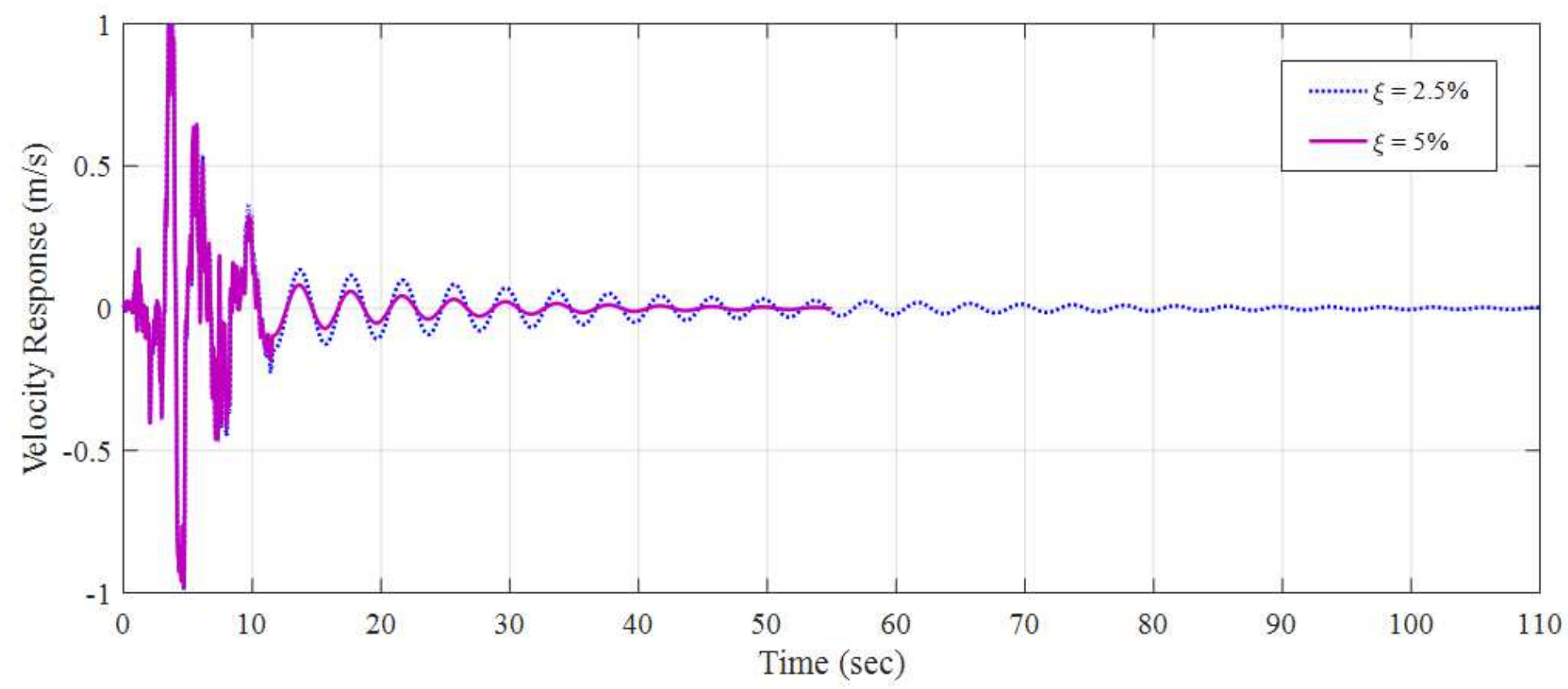

Figure 3

Effect of damping ratio on the amplitude of velocity response and the required time for the response to be fully damped

Force个

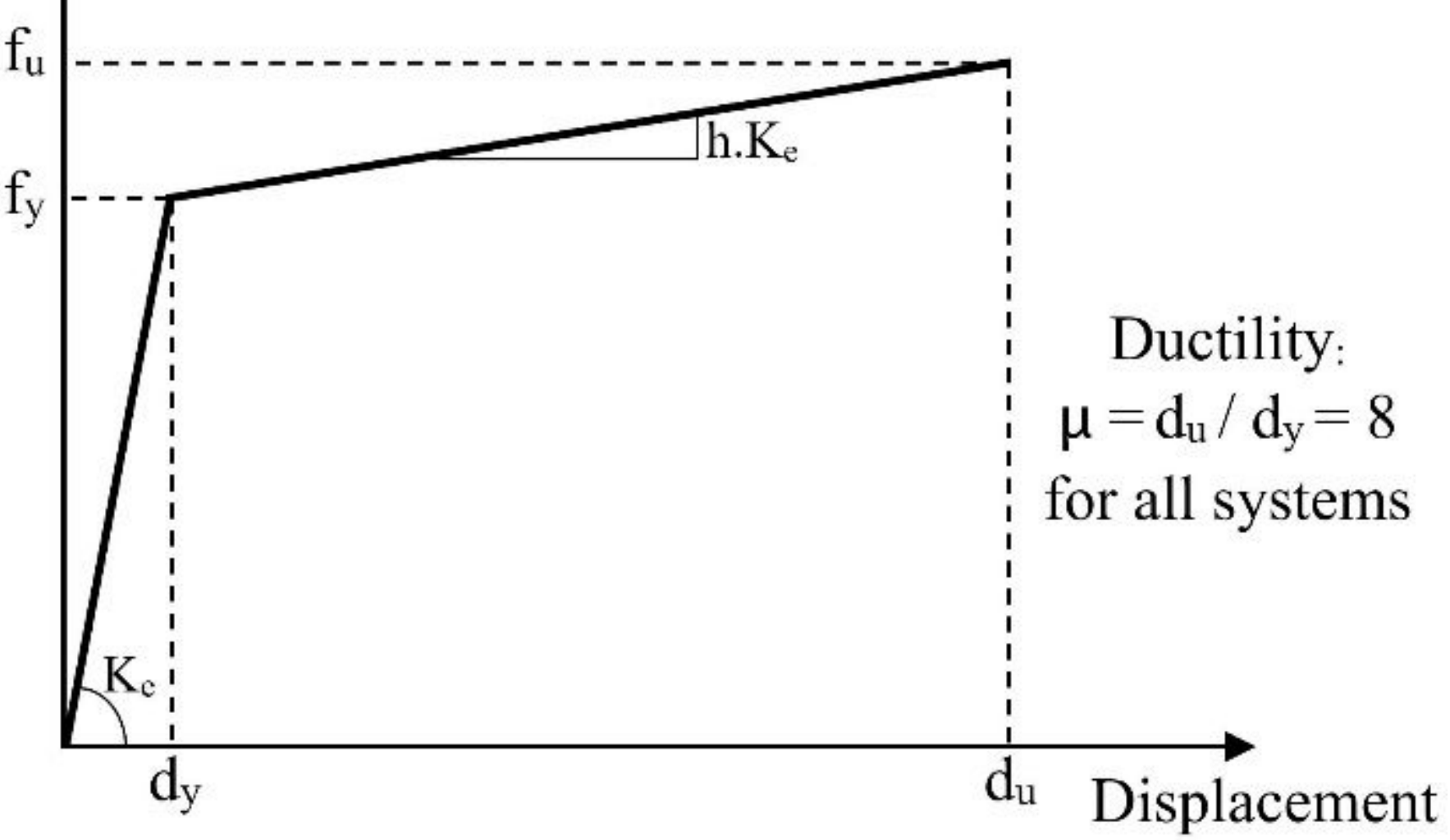

Figure 4 
Nonlinear behavior model of an SDOF system
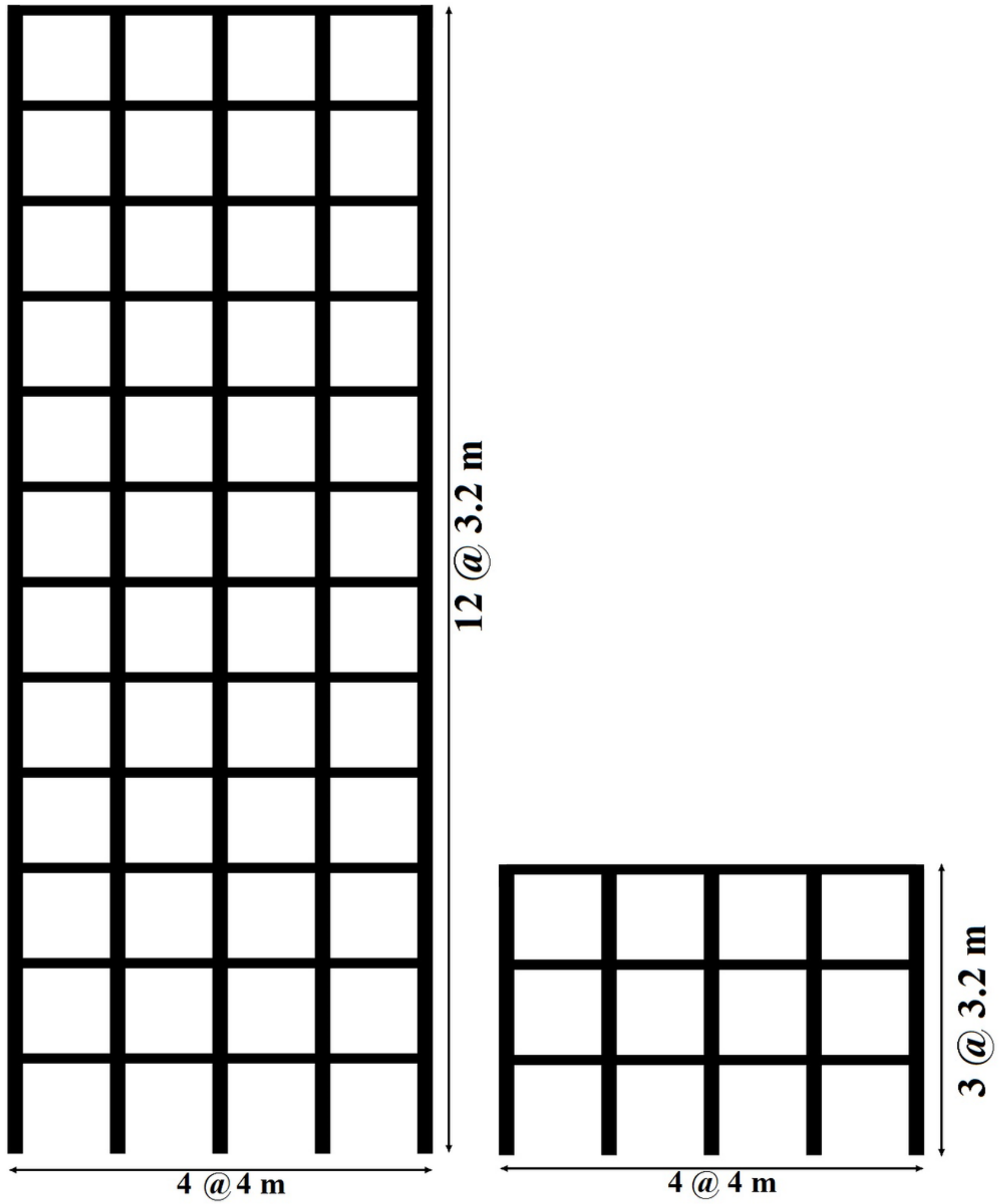

Figure 5

Elevation model of the steel frames 


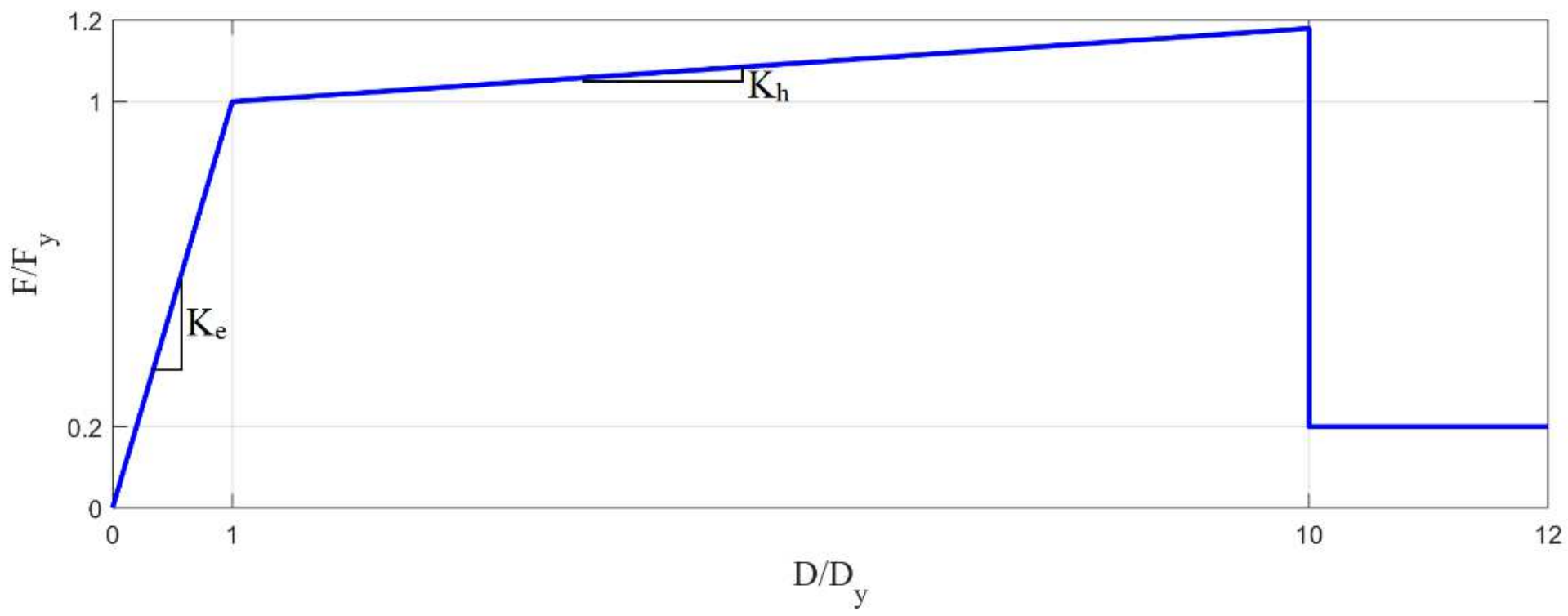

Figure 6

Bilinear model of FEMA beam and column (D: end rotation or axial deformation, F: end moment or axial force, Ke: initial elastic slope, Kh: strain hardening slope, $\mathrm{Kh} / \mathrm{Ke}=0.02$ )
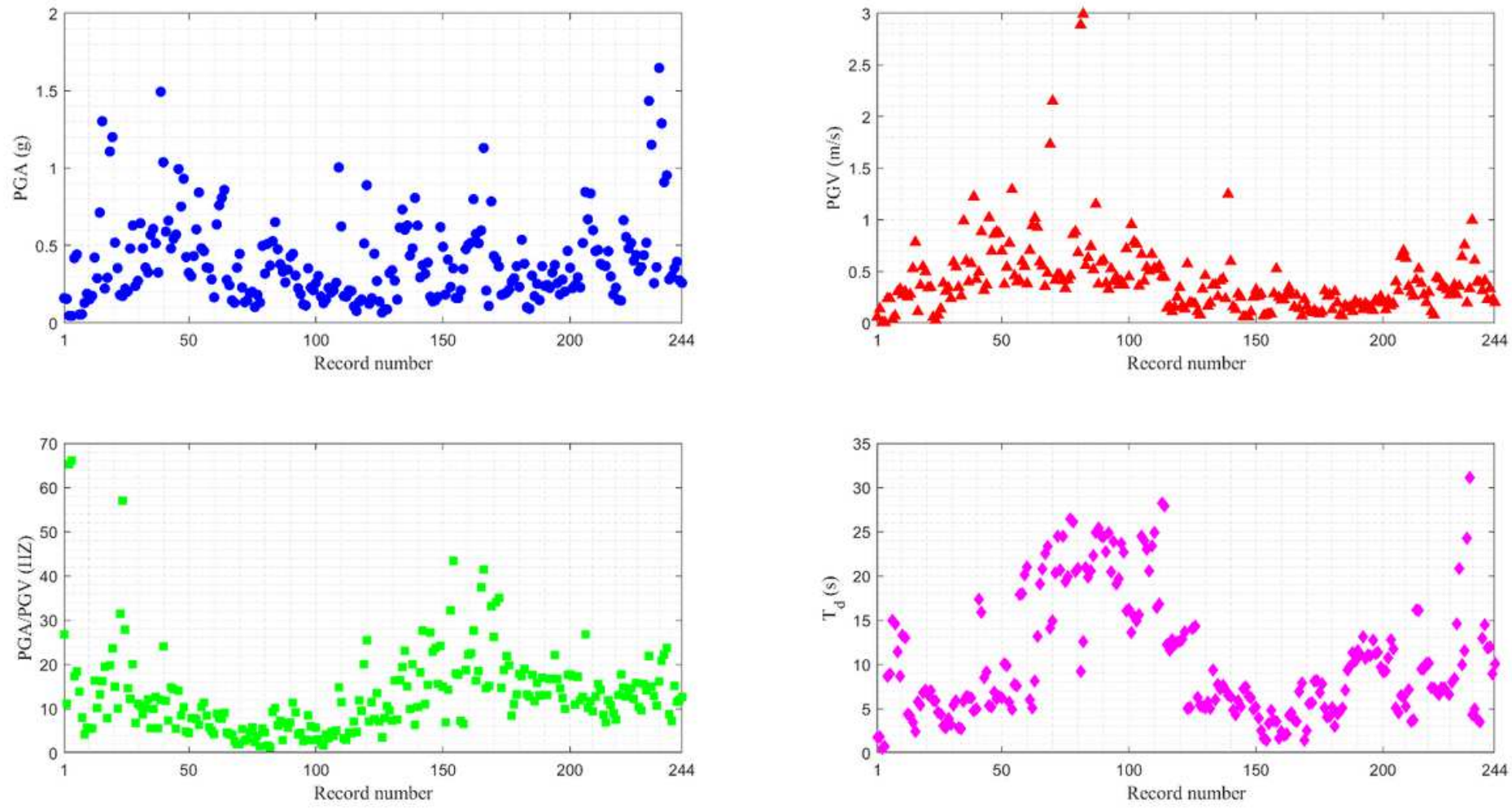

Figure 7

Descriptor parameters of selected records 

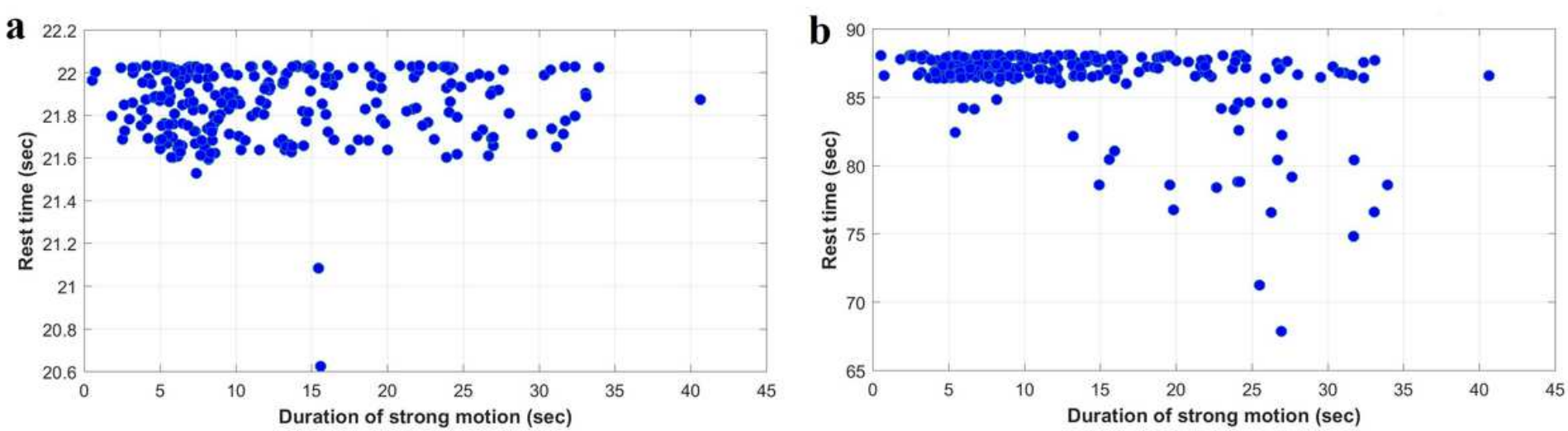

Figure 8

Evaluated rest time of the SDOF systems versus strong motion duration: (a) SDOF system with fundamental period $(T)$ of 1.0 sec., (b) SDOF system with $T=4.0 \mathrm{sec}$.
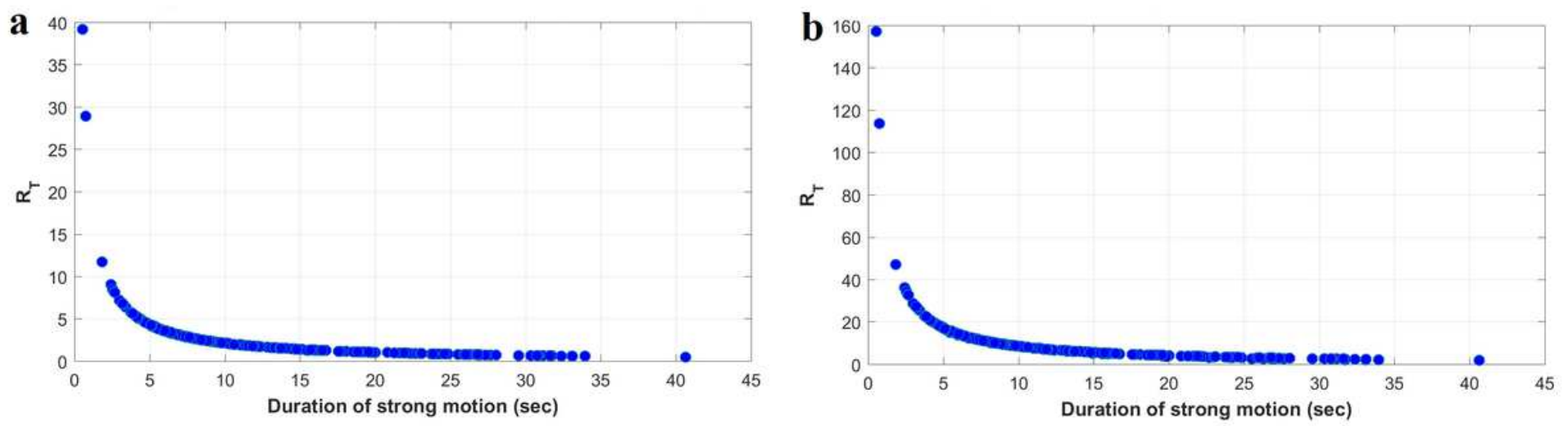

Figure 9

Normalized rest time (RT) of the SDOF systems versus strong motion duration: (a) SDOF system with $\mathrm{T}=$ 1.0 sec., (b) SDOF system with $T=4.0 \mathrm{sec}$.
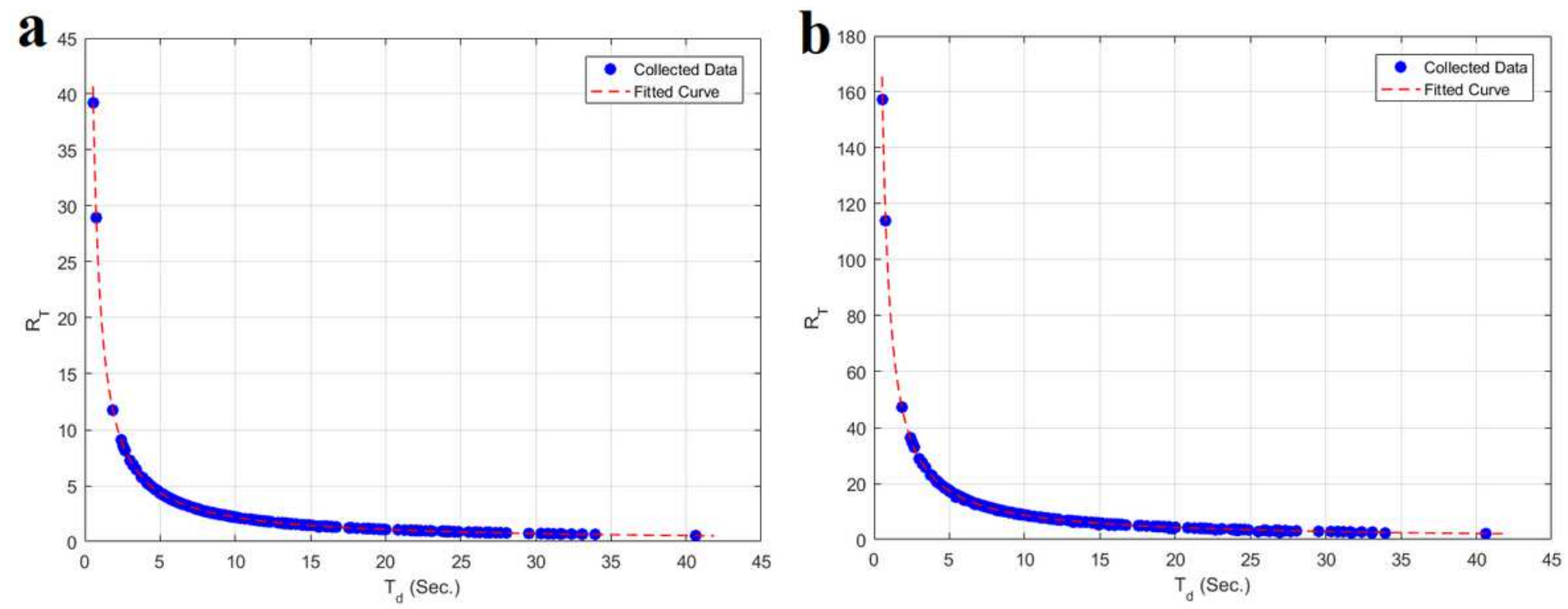
Curves fitted to the normalized rest time (RT) plotted versus the strong motion duration (Td): (a) for $T$ = $1.0 \mathrm{~s}$, (b) for $\mathrm{T}=4.0 \mathrm{~s}$
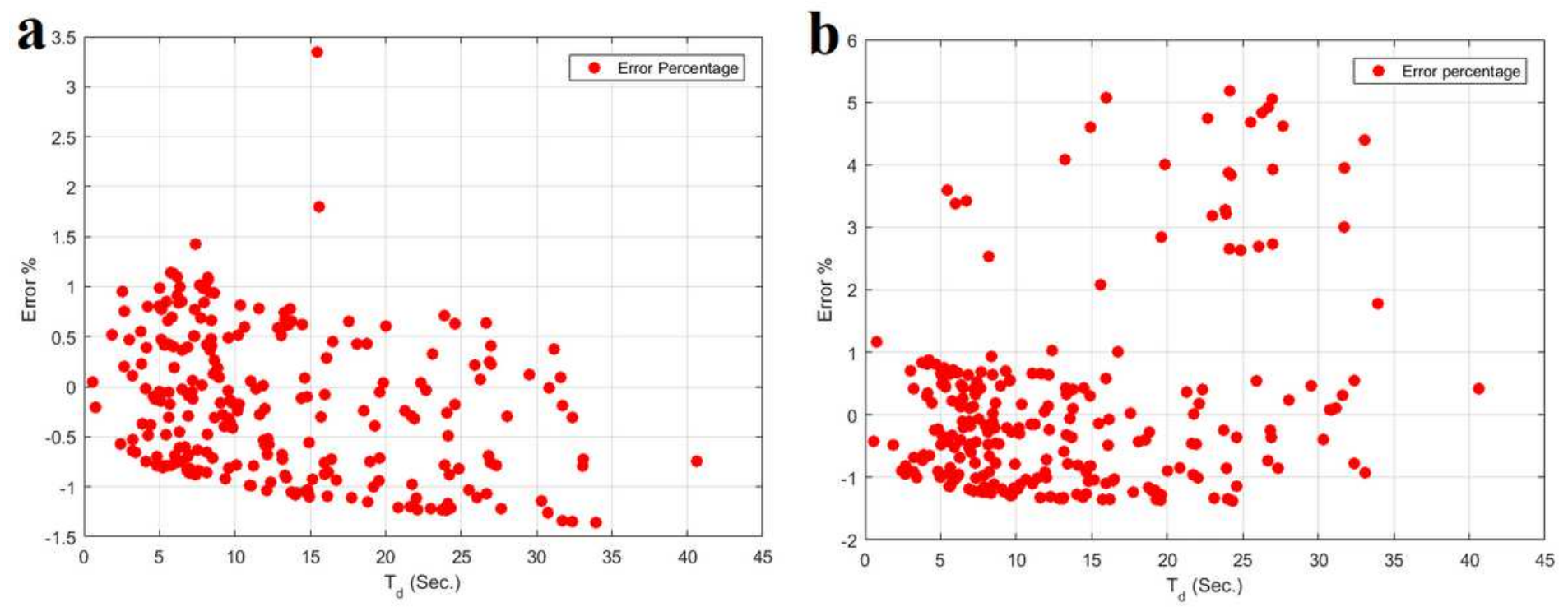

\section{Figure 11}

The percentage of difference between the collected rest time and the determined rest time obtained from the fitted curve's formula: (a) for $T=1.0 \mathrm{~s}$, (b) for $\mathrm{T}=4.0 \mathrm{~s}$
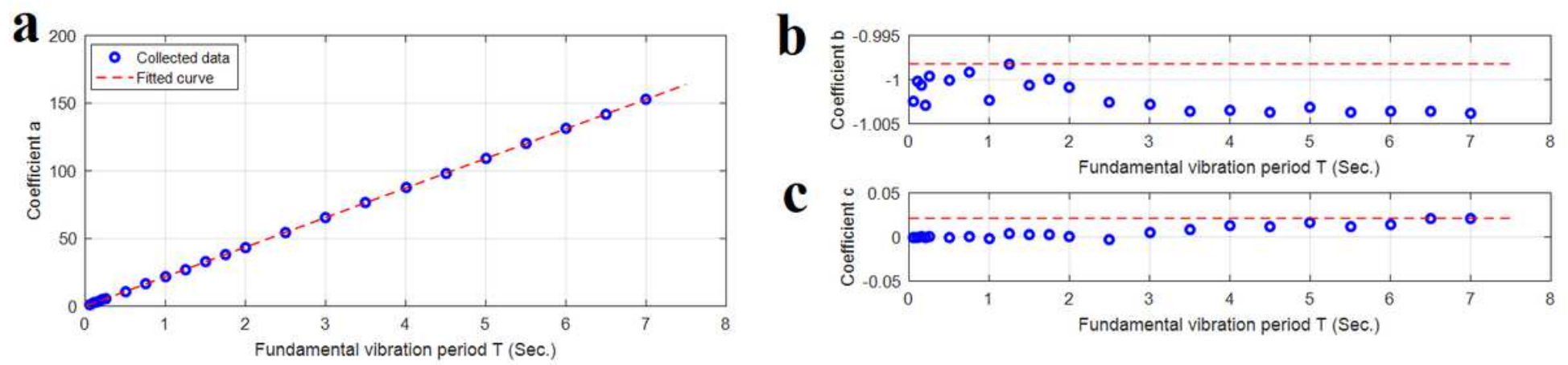

\section{Figure 12}

Curves fitted to the coefficients of the Eq. (3) plotted along with the obtained coefficients as a function of fundamental vibration period of SDOF systems: (a) for "a" coefficient, (b) for "b" coefficient, (c) for "c" coefficient 

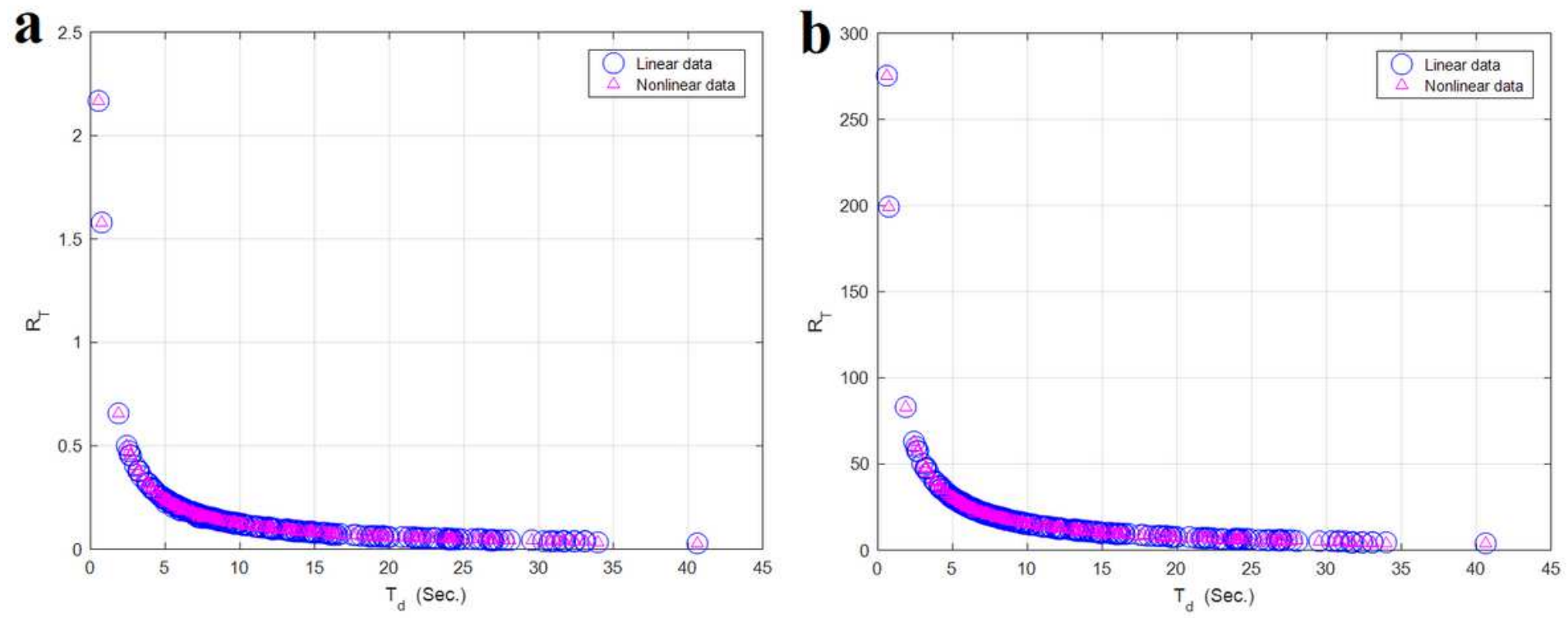

Figure 13

Normalized rest time (RT) plotted against strong motion duration (Td) for the collected data of a linear SDOF system as well as a nonlinear SDOF system: (a) for an SDOF with $\mathrm{T}=0.05 \mathrm{~s}$, (b) for an SDOF with $\mathrm{T}=7.0 \mathrm{~s}$
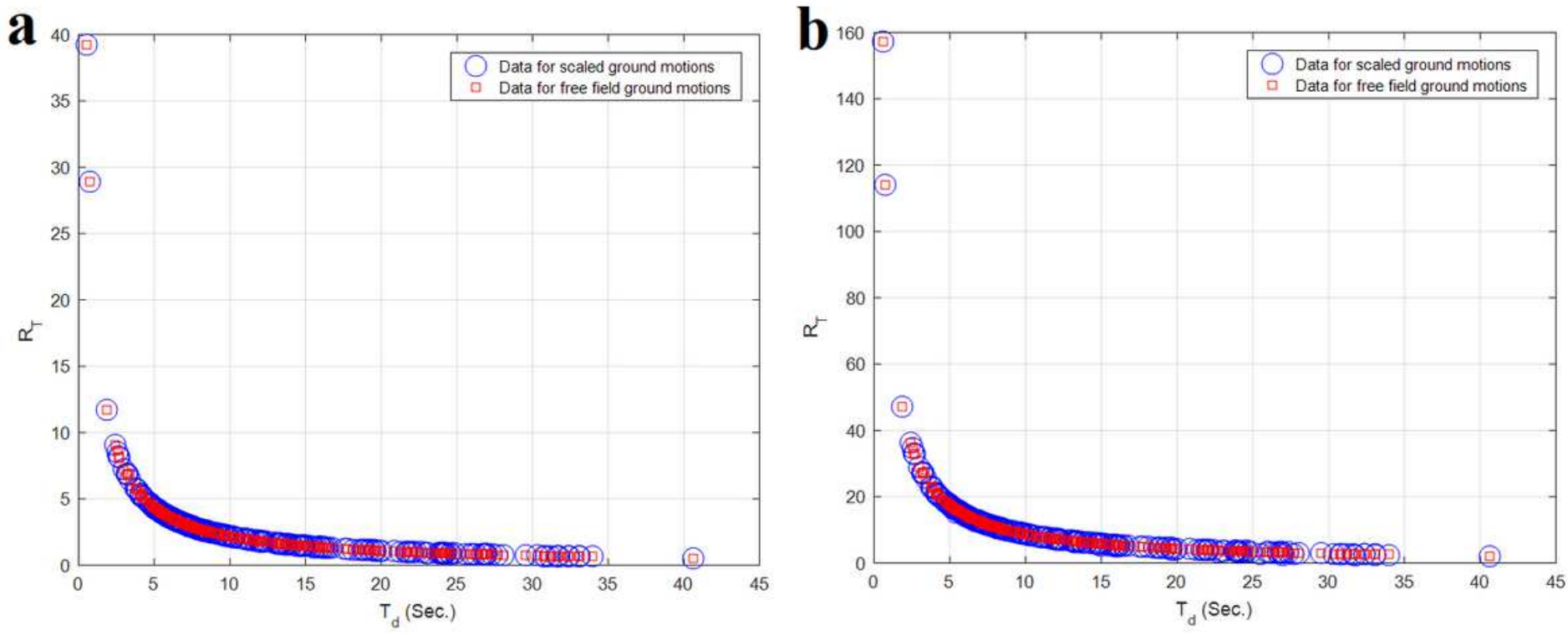

\section{Figure 14}

Normalized rest time (RT) plotted against strong motion duration (Td) While using scaled ground motions as well as free field ground motions: (a) for $T=1.0 \mathrm{~s}$, (b) for $\mathrm{T}=4.0 \mathrm{~s}$ 

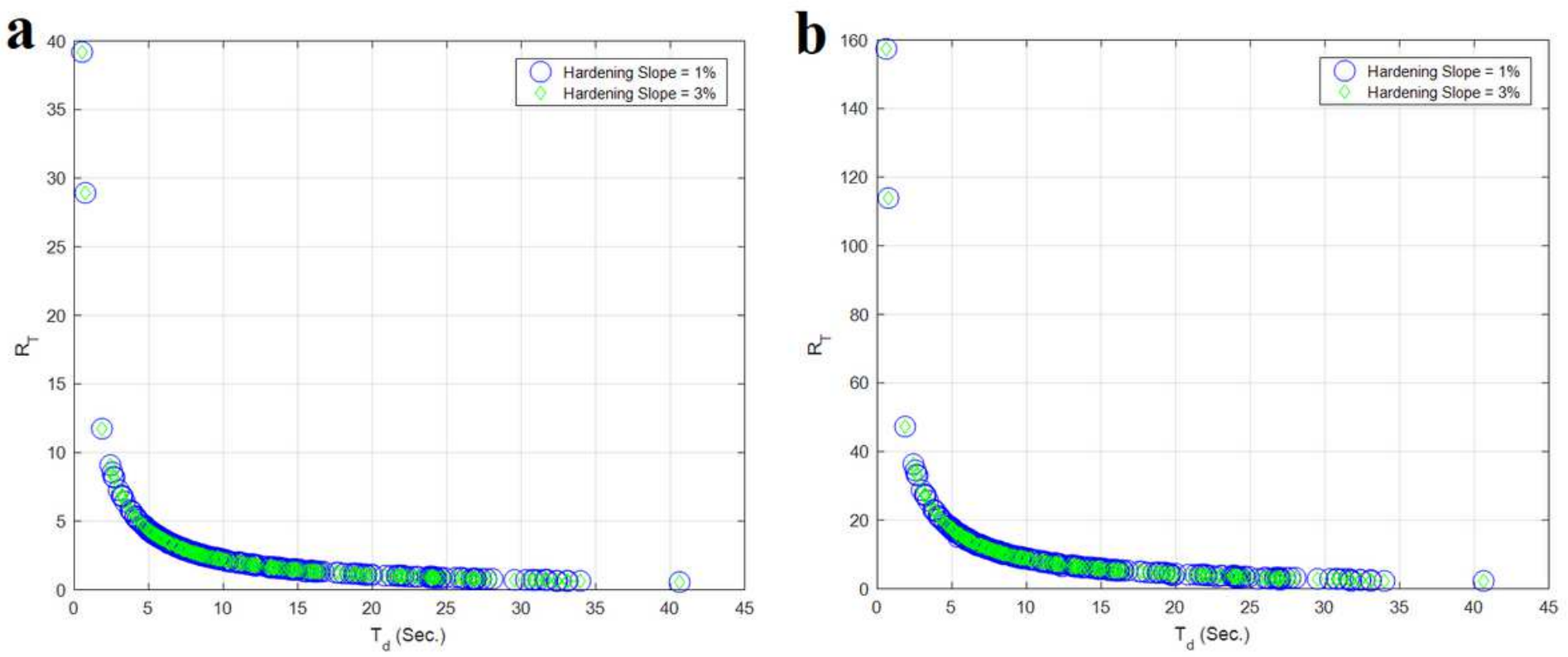

Figure 15

Normalized rest time (RT) plotted against the strong motion duration (Td) for different hardening slopes of nonlinear behavior: (a) for $\mathrm{T}=1.0 \mathrm{~s}$, (b) for $\mathrm{T}=4.0 \mathrm{~s}$
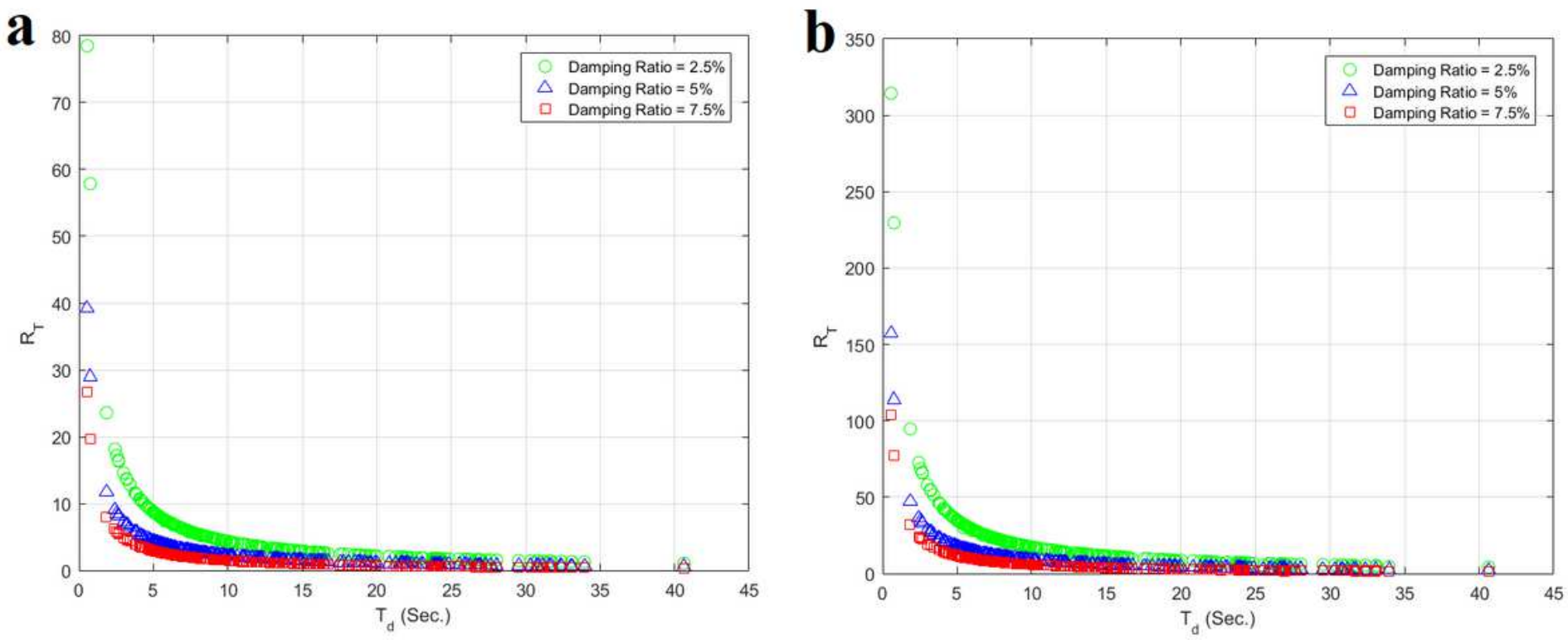

Figure 16

Normalized rest time (RT) plotted against the strong motion duration (Td) data series using different damping ratios: (a) for $T=1.0 \mathrm{~s}$, (b) for $\mathrm{T}=4.0 \mathrm{~s}$ 

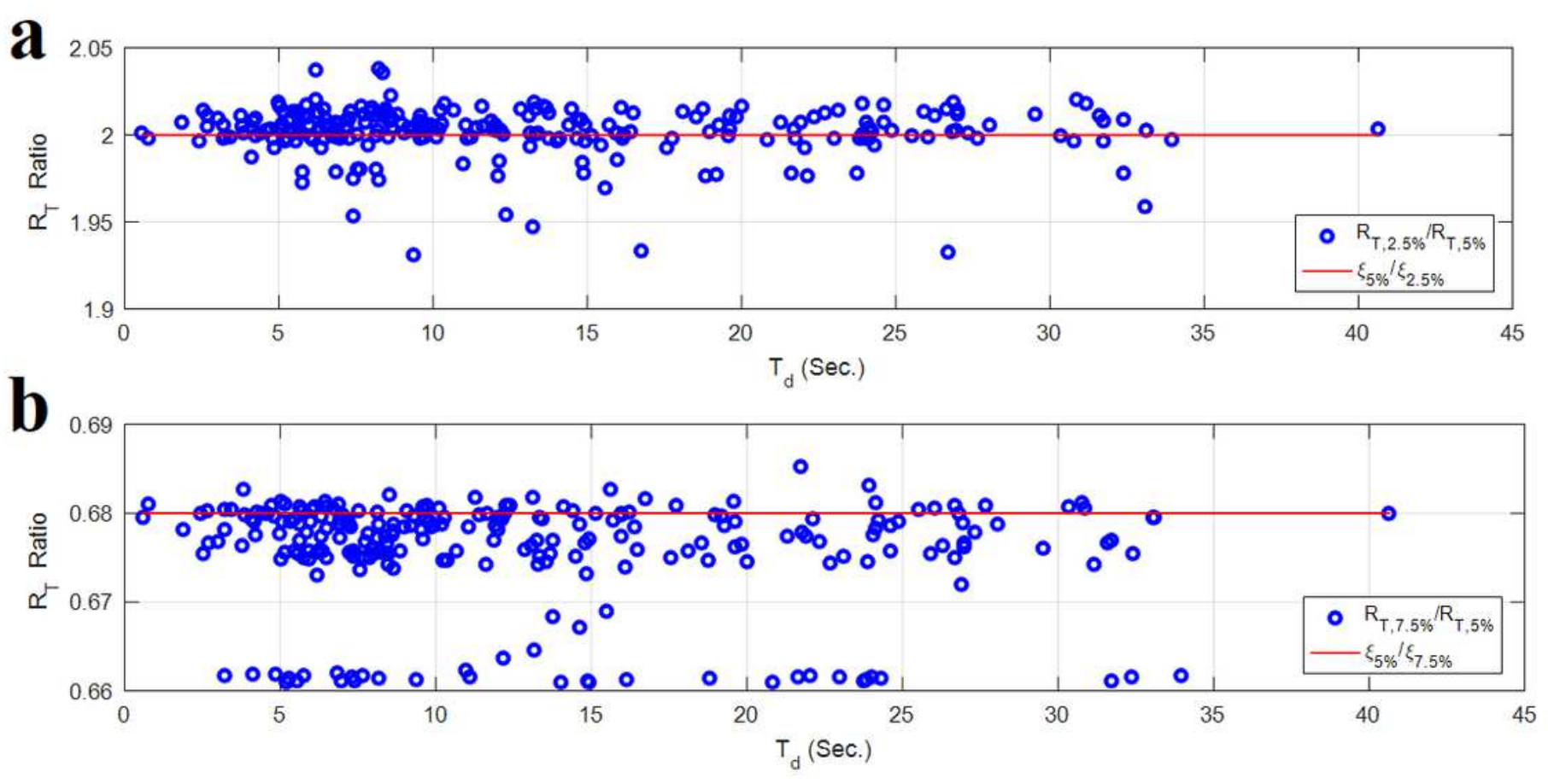

Figure 17

The ratio of normalized rest time (RT) versus strong motion duration (Td) for a SDOF having two different damping ratios with respect to the $5 \%$ damping ratio: (a) for $\xi=2.5 \%$, (b) for $\xi=7.5 \%$
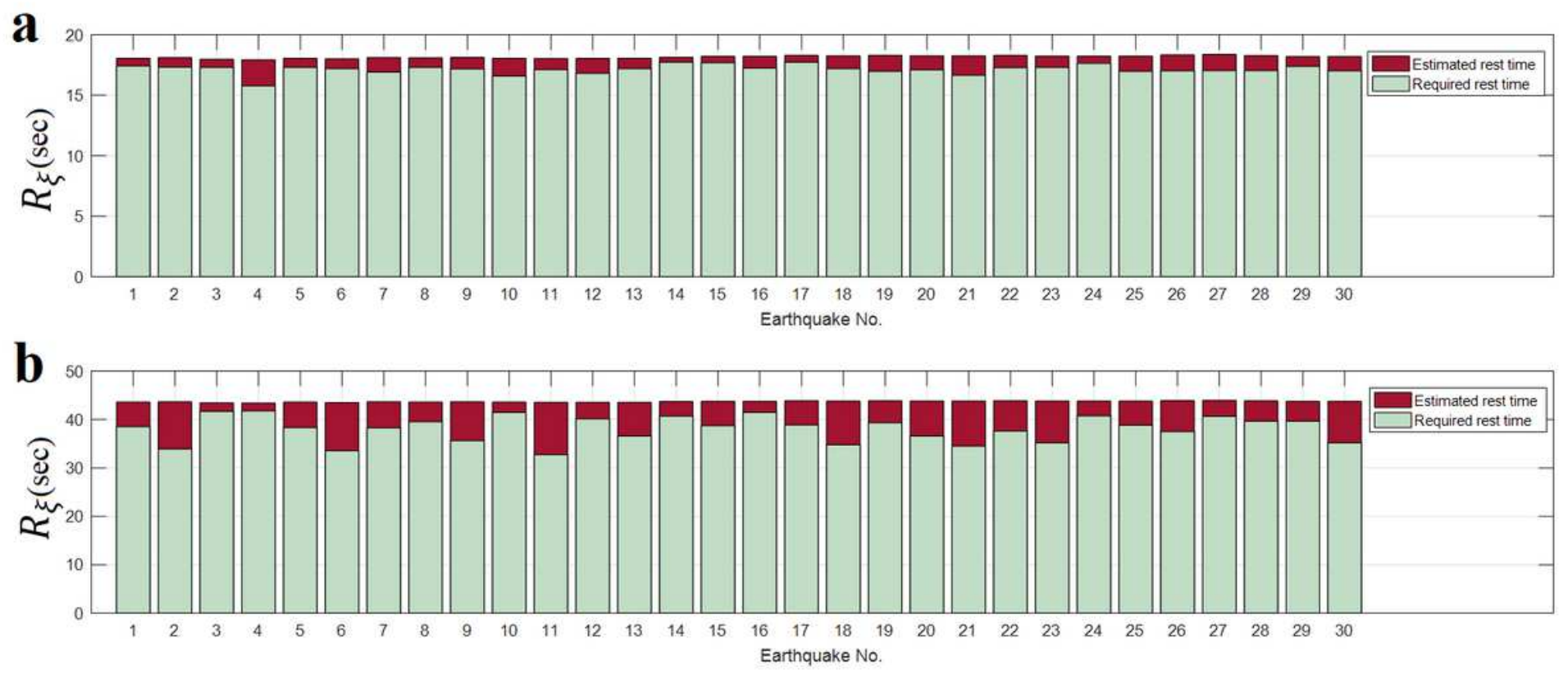

Figure 18

Required rest time obtained from the analysis versus the estimated rest time using the proposed formulation: (a) for the 3-story steel frame, (b) for the 12-story steel frame 

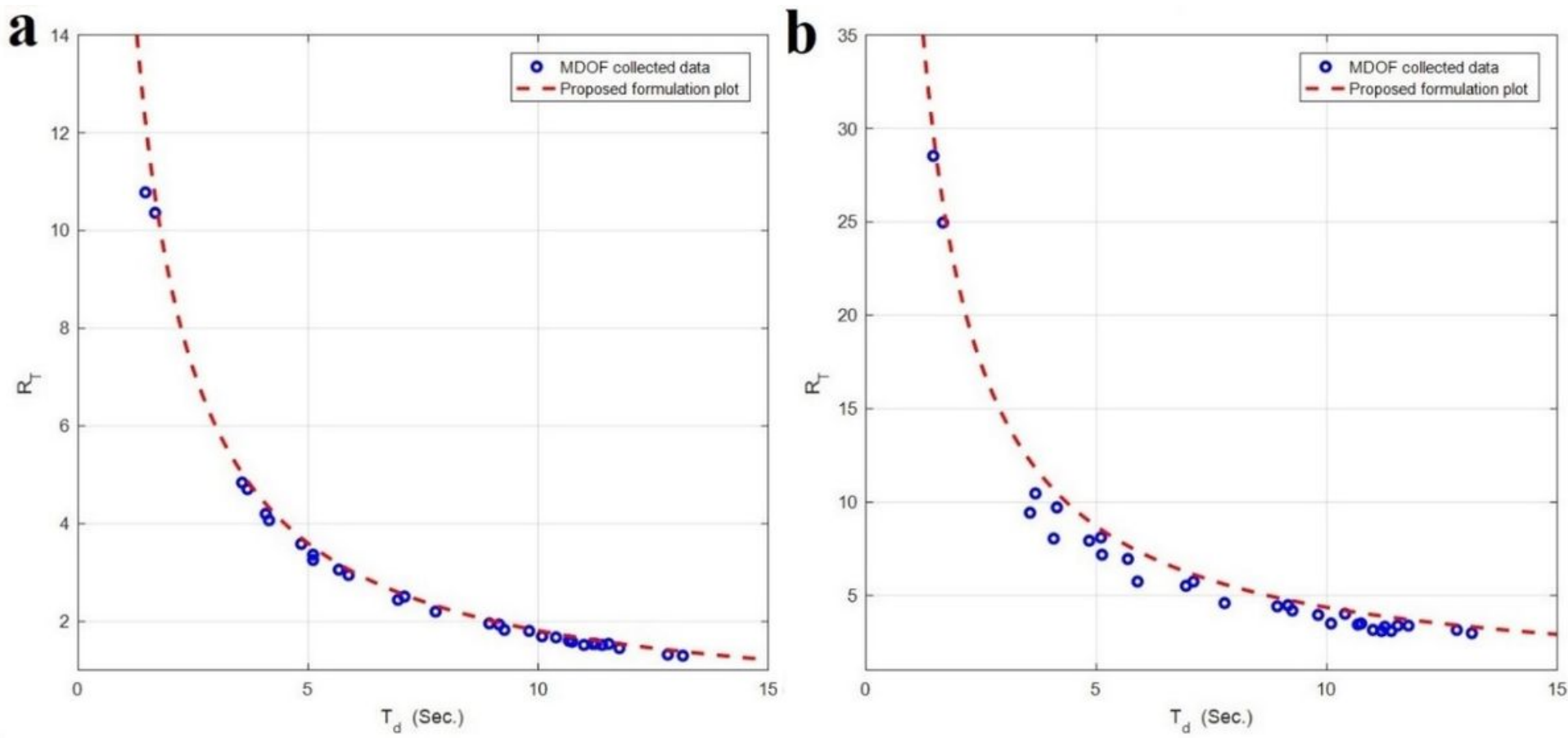

\section{Figure 19}

Normalized rest time (RT) plotted against the strong motion duration (Td) data series using different damping ratios: (a) for $\mathrm{T}=1.0 \mathrm{~s}$, (b) for $\mathrm{T}=4.0 \mathrm{~s}$

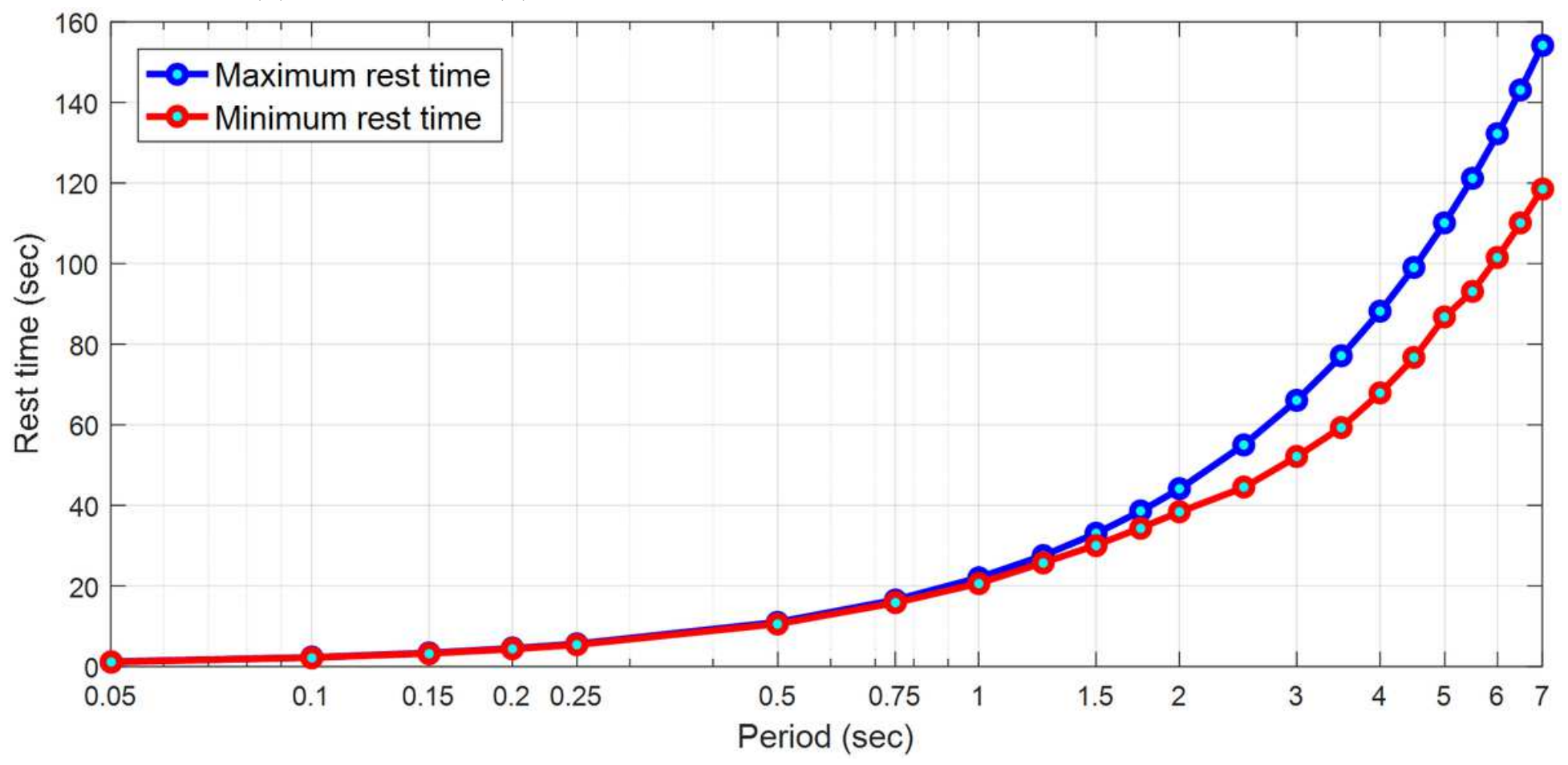

Figure 20

Maximum and minimum rest time plotted against the vibration period 